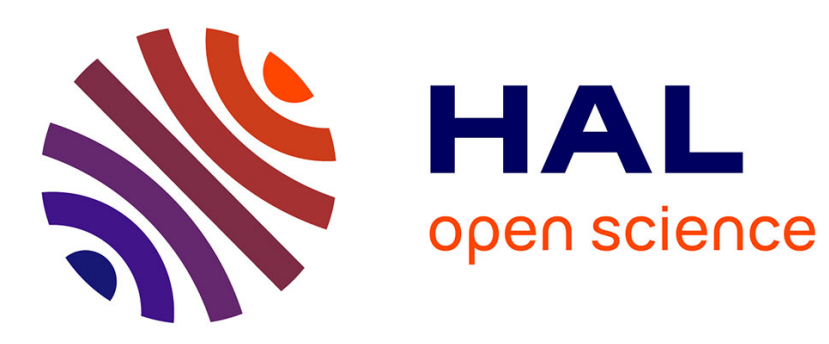

\title{
Opacity for Linear Constraint Markov Chains
}

Béatrice Bérard, Olga Kouchnarenko, John Mullins, Mathieu Sassolas

\section{To cite this version:}

Béatrice Bérard, Olga Kouchnarenko, John Mullins, Mathieu Sassolas. Opacity for Linear Constraint Markov Chains. [Research Report] LIP6 UMR 7606 UPMC Sorbonne Universités, France; Univ. de Franche-Comté; Ecole Polytechnique de Montréal; LACL, Université Paris-Est. 2016. hal-01384153

\section{HAL Id: hal-01384153 https://hal.sorbonne-universite.fr/hal-01384153}

Submitted on 19 Oct 2016

HAL is a multi-disciplinary open access archive for the deposit and dissemination of scientific research documents, whether they are published or not. The documents may come from teaching and research institutions in France or abroad, or from public or private research centers.
L'archive ouverte pluridisciplinaire HAL, est destinée au dépôt et à la diffusion de documents scientifiques de niveau recherche, publiés ou non, émanant des établissements d'enseignement et de recherche français ou étrangers, des laboratoires publics ou privés. 


\title{
Opacity for Linear Constraint Markov Chains ${ }^{\star}$
}

\author{
Béatrice Bérard ${ }^{1}$ and Olga Kouchnarenko ${ }^{2}$ and John Mullins ${ }^{3}$ and \\ Mathieu Sassolas ${ }^{4}$ \\ 1 Sorbonne Université, UPMC Univ Paris 06, UMR 7606, LIP6, Paris, France \\ 2 Université de Franche-Comté, FEMTO-ST, CNRS UMR 6174, Inria/NGE, \\ Besançon, France \\ 3 Dept. of Computer \& Software Eng., École Polytechnique de Montréal \\ Montreal (Quebec), Canada, H3C 3A7 \\ 4 Université Paris-Est, LACL, Créteil, France
}

\begin{abstract}
On a partially observed system, a secret $\varphi$ is opaque if an observer cannot ascertain that its trace belongs to $\varphi$. We consider specifications given as Constraint Markov Chains (CMC), which are underspecified Markov chains where probabilities on edges are required to belong to some set. The nondeterminism is resolved by a scheduler, and opacity on this model is defined as a worst case measure over all implementations obtained by scheduling. This measures the information obtained by a passive observer when the system is controlled by the smartest scheduler in coalition with the observer. When restricting to the subclass of Linear CMC, we compute (or approximate) this measure and prove that refinement of a specification can only improve opacity.
\end{abstract}

\section{Introduction}

Context and motivation. When modeling complex systems, a top-down approach allows gradually specifying various system requirements, while preserving some behavioral properties, like safety, reachability, and liveness under some conditions.

Security requirements, which are not behavioral ones [1], may not fare well under refinement, unless tailored specially to do so, as in [2]. Several known security properties such as noninference or anonymity can be encoded in the framework of opacity [3,4,2]. In this context, an external observer tries to discover whether a predicate (given as an $\omega$-regular set) holds by partially observing the system through a projection of its actions.

\footnotetext{
* Partially supported by a grant from Coopération France-Québec, Service Coopération et Action Culturelle 2012/26/SCAC (French Government), the NSERC Discovery Individual grant No. 13321 (Government of Canada), the FQRNT Team grant No. 167440 (Quebec's Government) and the CFQCU France-Quebec Cooperative grant No. 167671 (Quebec's Government). This research has been partially done while this author was visiting the LIP6, Université Pierre \& Marie Curie.
} 
A system is opaque if the attacker fails to discover this information. In the possibilistic setting, a violation of opacity captures the existence of at least one perfect leak.

In probabilistic models like Discrete Time Markov Chains (DTMCs), naturally random events such as faults or message transmission failure, can be taken into account. Opacity was extended in this setting [5,67] to provide various measures of what is disclosed by observation.

Consider for instance the two systems in Fig. 1(a) (b), which are DTMCs with the addition of labels on states (indicated inside). We assume that the occurrence of $b$ must be kept secret and that all labels except $b$ are observable. In this case, the only runs disclosing the secret are those observed by $a d^{\omega}$, since every such run betrays the occurrence of $b$. The probability of disclosure is $1 / 4$ in $\mathcal{A}_{1}$ while it is $3 / 4$ in $\mathcal{A}_{2}$, hence $\mathcal{A}_{1}$ is more secure than $\mathcal{A}_{2}$. Our aim is to establish sufficient conditions on systems like $\mathcal{A}_{1}$ and $\mathcal{A}_{2}$, that can be compared, for one of them to be more secure than the other.

In the process of system modeling, it is common practice to use underspecified models as first steps of specification. A first approach is to con-

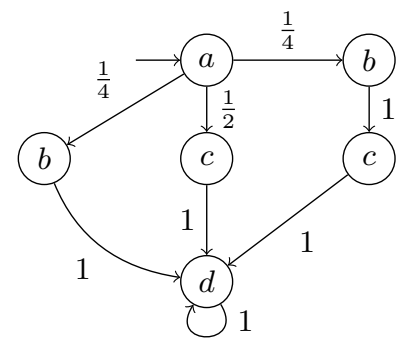

(a) $\mathcal{A}_{1}$

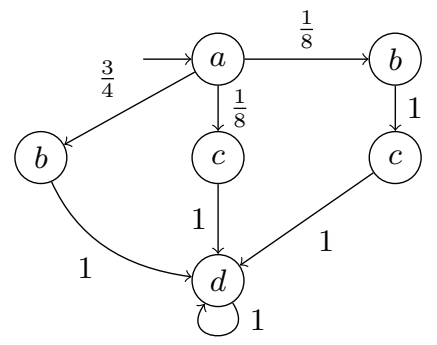

(b) $\mathcal{A}_{2}$

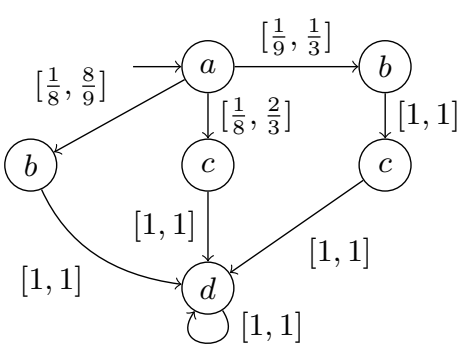

(c) $\mathcal{S}$

Fig. 1. Probabilistic systems $\mathcal{A}_{1}$ or $\mathcal{A}_{2}$ implementing underspecified system $\mathcal{S}$. 
sider sub-stochastic models where transition probabilities need not sum up to 1 . In this framework, the notions of satisfaction and simulation were extensively studied in [8]. The second approach is to introduce nondeterminism in the model to describe environment choices [9/0/11/2/3]7/14. These models have also been studied in relation to the refinement process [9], with the particular case of Interval Markov Chains (IMCs) where the transitions are equipped with probability bounds in the form of intervals, as done in Fig. 1(c), For example, both systems of Fig. 1(a) (b) could have been derived from the single underspecified system $\mathcal{S}$ of Fig. 1(c), with the same structure but imprecise probabilities.

Unfortunately, while closure under conjunction is a nice feature for specification formalisms, IMCs do not have this property. This is shown in [12, where Constraint Markov Chains (CMCs), first introduced in [9], are considered for the specification of finite state processes, and proved to provide a more robust model. In a CMC, the family of intervals associated with a state is replaced by a given (possibly infinite) set of distributions.

Scheduling is an effective way to obtain implementations of a CMC: at each step, a scheduler provides a distribution belonging to the given set, thus producing a (possibly infinite) DTMC on-the-fly. In the case of opacity, a scheduler represents a strategy of an agent inside the system, trying to disclose as much information as possible to a passive observer. Several works used schedulers to evaluate disclosure: [7] in the context of (fully specified) Markov Decision Processes and [15] for IMCs.

Contributions. We extend the latter work, investigating opacity for CMCs. As before, disclosure is defined in the worst case scenario, as the supremum of the disclosure for all scheduled implementations. This measures the information obtained by a passive observer when the system is controlled by the smartest scheduler in coalition with the observer.

However, without an explicit description of the given probability sets, algorithmic questions cannot be solved on CMCs. Therefore, we consider here a subclass called Linear Constraint Markov Chains (LCMCs), where the set of distributions associated with a state is defined by linear inequalities. This class is closed under conjunction and contains IMCs.

We first show how to compute the disclosure for a subclass of LCMCs, where no transition can be completely blocked by the scheduler. In the general case, we give an overapproximation for bounded memory schedulers. We then establish monotonicity of the disclosure for LCMCs: refining an LCMC can only improve the opacity of all implementations obtained by scheduling. 
Organization of the paper. After short preliminaries on probabilistic transition systems and opacity (Section 2), we recall in Section 3 the background on Constraint Markov Chains, with the associated refinement relations, and we define probabilistic disclosure in this contex. We show how to compute this measure for a restricted case of LCMCs in Section 4, with an approximation scheme for the general case. Finally, we prove monotonicity of opacity under refinement in Section 5 .

\section{Probabilistic transition systems and opacity}

The set of natural numbers is denoted by $\mathbb{N}$ and the set of rational numbers by $\mathbb{Q}$. The composition of relations $\mathcal{R}_{2}$ and $\mathcal{R}_{1}$ is defined by $\mathcal{R}_{2} \circ \mathcal{R}_{1}=\left\{(x, z) \mid \exists y,(x, y) \in \mathcal{R}_{1} \wedge(y, z) \in \mathcal{R}_{2}\right\}$. Given a finite alphabet $\Sigma$, we denote by $\Sigma^{*}$ (resp. $\Sigma^{\omega}$ ) the set of finite (resp. infinite) words over $\Sigma$, with $\Sigma^{\infty}=\Sigma^{*} \cup \Sigma^{\omega}$ and $\varepsilon$ the empty word. We denote by $|w|$ the length of word $w$ in $\mathbb{N} \cup\{+\infty\}$ with the same notation $|E|$ for the cardinality of a set $E$.

Given a countable set $Z$, a discrete distribution is a mapping $\mu: Z \rightarrow$ $[0,1]$ such that $\sum_{z \in Z} \mu(z)=1$. The support of $\mu$ is $\operatorname{supp}(\mu)=\{z \in$ $Z \mid \mu(z)>0\}$. The set of all discrete distributions on $Z$ is denoted by $\operatorname{Dist}(Z)$. When dealing with a joint distribution $\mu$ on domain $Z_{1} \times Z_{2}$, we write $\mu\left(Y_{1}, Y_{2}\right)=\sum_{y_{1} \in Y_{1}, y_{2} \in Y_{2}} \mu\left(y_{1}, y_{2}\right)$ for $Y_{1} \subseteq Z_{1}$ and $Y_{2} \subseteq Z_{2}$, and we use as shorthands $\mu\left(y_{1}, Y_{2}\right)=\mu\left(\left\{y_{1}\right\}, Y_{2}\right)$ and $\mu\left(Y_{1}, y_{2}\right)=\mu\left(Y_{1},\left\{y_{2}\right\}\right)$.

\subsection{Probabilistic Labelled Transition Systems and their languages}

The secret to be protected from disclosure is described by a Deterministic Parity Automaton (DPA, see example in Fig. 2). Implementations are given by Probabilistic Transition Systems (PTSs). They are classical Discrete Time Markov Chains, with the addition of state labeling [8], restricting the processes of [9] with a countable set of states.

Definition 2.1 (Deterministic Parity Automaton). A deterministic parity automaton (DPA) over finite alphabet $\Sigma$ is a tuple $\mathcal{A}=\left(Q, q_{0}, \theta, F\right)$, where $Q$ is a finite set of states, $q_{0} \in Q$ is an initial state, $\theta: Q \times \Sigma \rightarrow Q$ is a transition function, and $F$ is a mapping from $Q$ to a finite set of colors $\{1, \ldots, k\}$.

A run of $\mathcal{A}$ on a word $w=a_{1} a_{2} \cdots \in \Sigma^{\omega}$ is an infinite sequence $\rho=q_{0} q_{1} \cdots \in Q^{\omega}$ such that for all $i \geq 0, q_{i+1}=\theta\left(q_{i}, a_{i+1}\right)$. For such a 


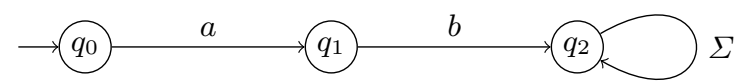

Fig. 2. A DPA for $\varphi_{b}=a b \Sigma^{\omega}$ with $F\left(q_{0}\right)=F\left(q_{2}\right)=1$ and $F\left(q_{2}\right)=2$.

run $\rho$, we define $\operatorname{Inf}(\rho)$ as the set of states appearing infinitely often in the sequence. The run is accepting if $\min \{F(q) \mid q \in \operatorname{Inf}(\rho)\}$ is even. In this case, the corresponding word is accepted by $\mathcal{A}$ and $\mathcal{L}(\mathcal{A})$ is the subset of $\Sigma^{\omega}$ of words accepted by $\mathcal{A}$. A subset $K$ of $\Sigma^{\omega}$ is $\omega$-regular if there is an automaton $\mathcal{A}$ such that $K=\mathcal{L}(\mathcal{A})$.

Definition 2.2 (Probabilistic Transition Systems). A probabilistic transition system (PTS) over alphabet $\Sigma$ is a tuple $\mathcal{A}=\left(Q, q_{\text {init }}, \Delta, L\right)$ where $Q$ is a countable set of states, with $q_{\text {init }} \in Q$ the initial state, $\Delta: Q \rightarrow \operatorname{Dist}(Q)$ is a mapping associating with any state $q \in Q$ a distribution $\Delta(q)$ over $Q$, with finite support, and $L: Q \rightarrow \Sigma$ is the labeling function on states.

PTSs can be seen as Discrete Time Markov Chains where the states are equipped with labels in $\Sigma$. While our presentation uses an alphabet $\Sigma$ of labels for simplicity, it is always possible as done in [912] to consider the case where $\Sigma=2^{A}$ for a set $A$ of atomic propositions.

A (finite or infinite) run of $\mathcal{A}$ starting from state $q \in Q$ is a sequence of states $\rho=q_{0} q_{1} q_{2} \ldots$ such that $q_{0}=q$ and for each $i, 0 \leq i<|\rho|$, $\Delta\left(q_{i}\right)\left(q_{i+1}\right)>0$. When the run is finite $\rho=q_{0} q_{1} \ldots q_{n}$, we note $q_{n}=\operatorname{lst}(\rho)$. The trace of $\rho$ is the word $\operatorname{tr}(\rho)=L\left(q_{0}\right) L\left(q_{1}\right) \ldots \in \Sigma^{\infty}$. We denote by $\operatorname{Runs}_{q}(\mathcal{A})$ the set of infinite runs starting from $q$ and we set $\operatorname{Runs}(\mathcal{A})=$ Runs $_{q_{\text {init }}}(\mathcal{A})$, and $\operatorname{Tr}(\mathcal{A})=\{\operatorname{tr}(\rho) \mid \rho \in \operatorname{Runs}(\mathcal{A})\}$, the set of traces of $\mathcal{A}$. We also define $F$ Runs $_{q}(\mathcal{A})$ the set of finite runs starting from $q$, and similarly $F \operatorname{Runs}(\mathcal{A})=$ FRuns $_{q_{\text {init }}}(\mathcal{A})$ and $F \operatorname{Tr}(\mathcal{A})=\{\operatorname{tr}(\rho) \mid \rho \in$ FRuns $(\mathcal{A})\}$, the subset of $\Sigma^{*}$ of finite traces of $\mathcal{A}$.

Recall [16] that a probability measure $\mathbf{P}_{\mathcal{A}}$ can be defined on $\operatorname{Runs}(\mathcal{A})$ : measurable sets are generated by cones, where the cone $C_{\rho}$ associated with a finite run $\rho=q_{0} q_{1} \ldots q_{n}$ is the subset of infinite runs in $\operatorname{Runs}(\mathcal{A})$ having $\rho$ as prefix. The probability of $C_{\rho}$ is $\mathbf{P}_{\mathcal{A}}\left(C_{\rho}\right)=\prod_{i=0}^{n-1} \Delta\left(q_{i}\right)\left(q_{i+1}\right)$. The cone of a word $w \in \Sigma^{*}$ is defined by $C_{w}=\bigcup_{\rho \in \operatorname{tr}^{-1}(w)} C_{\rho}$. 


\section{$2.2 \quad$ Probabilistic Opacity}

The original definition of opacity was given in [4] for (non probabilistic) transition systems, w.r.t. some observation function $\mathcal{O}$ and some predicate $\varphi$ (the secret) on the runs of the system.

For an $\omega$-regular set $\varphi \subseteq \Sigma^{\omega}$, a run $\rho$ of a PTS $\mathcal{A}$ satisfies $\varphi$ if its trace belongs to $\varphi$. We consider an observation function defined as a morphism $\mathcal{O}: \Sigma^{\infty} \rightarrow \Sigma_{o}^{\infty}$, based on a mapping $\pi: \Sigma \rightarrow \Sigma_{o} \cup\{\varepsilon\}$ for a finite alphabet $\Sigma_{o}$. For instance, if $\Sigma=2^{A}$ for a set $A$ of atomic propositions, we can define a subset $A_{o}$ of observable propositions. Then, by setting $A_{u}=A \backslash A_{o}, \Sigma_{o}=2^{A_{o}}, \pi$ can be defined by $\pi(\sigma)=\varepsilon$ for $\sigma \in 2^{A_{u}}$ and $\pi(\sigma)=\sigma \cap A_{o}$ otherwise.

The set $\varphi$ is opaque with respect to $\mathcal{A}$ and $\mathcal{O}$ if each time a word satisfies $\varphi$, another word with the same observation does not. More precisely, the set of words violating this condition is defined by $\mathcal{V}(\mathcal{A}, \mathcal{O}, \varphi)=$ $(\operatorname{Tr}(\mathcal{A}) \cap \varphi) \backslash\left(\mathcal{O}^{-1}(\mathcal{O}(\operatorname{Tr}(\mathcal{A}) \backslash \varphi))\right)$ and $\varphi$ is opaque if $\mathcal{V}(\mathcal{A}, \mathcal{O}, \varphi)=\emptyset$.

This set is used in [175] to define various notions of probabilistic opacity. For instance, in [5], one of the measures corresponds to the particular case where $\varphi$ is the set of finite traces of runs reaching a fixed set of secret states. The boolean property is extended by defining the probability of this set, which is measurable since $\varphi$ is $\omega$-regular:

Definition 2.3 (Probabilistic Disclosure). Let $\mathcal{A}$ be a PTS, $\mathcal{O}$ an observation function and $\varphi$ an $\omega$-regular predicate. The probabilistic disclosure of $\varphi$ in $\mathcal{A}$ for $\mathcal{O}$ is $\operatorname{Disc}(\mathcal{A}, \mathcal{O}, \varphi)=\mathbf{P}_{\mathcal{A}}(\mathcal{V}(\mathcal{A}, \mathcal{O}, \varphi))$.

For instance, recall systems $\mathcal{A}_{1}$ and $\mathcal{A}_{2}$ of Fig. 1. The secret predicate in this case is the set $\varphi_{b}=a b \Sigma^{\omega}$, accepted by the DPA in Fig. 2, and the observation function is the projection $\pi$ onto $\{a, c, d\}^{\omega}$. This predicate is not opaque since the run $a b d^{\omega}$ discloses the occurrence of $b$. This is measured by the disclosure: $\operatorname{Disc}\left(\mathcal{A}_{1}, \pi, \varphi_{b}\right)=\mathbf{P}_{\mathcal{A}_{1}}\left(a b d^{\omega}\right)=\frac{1}{4}$ and $\operatorname{Disc}\left(\mathcal{A}_{2}, \pi, \varphi_{b}\right)=\mathbf{P}_{\mathcal{A}_{2}}\left(a b d^{\omega}\right)=\frac{3}{4}$.

Remark that disclosure only measures probabilities of the observer being sure that the run is in the secret. For example, one can model anonymity of an agent $\alpha$ initiating some protocol by defining $\varphi_{\alpha}$ as the set of all runs initiated by $\alpha$. Anonymity of $\alpha$ is then equivalent to opacity of $\varphi_{\alpha}$. In the case where anonymity is not guaranteed, disclosure provides a measure of the threat. In the case where anonymity holds, this measure will be 0 and does not give any insight on the "strength" of anonymity. Other notions measuring this strength were proposed in [18,19] and quantitative opacity for partial disclosure of the secret have also been defined 
in [6], although they are not linear hence do not fare well under standard optimization techniques.

For two PTSs $\mathcal{A}_{1}$ and $\mathcal{A}_{2}$ over the same alphabet $\Sigma$, predicate $\varphi$ and observation function $\mathcal{O}$, we say that $\mathcal{A}_{1}$ is more opaque than $\mathcal{A}_{2}$ if $\operatorname{Disc}\left(\mathcal{A}_{1}, \mathcal{O}, \varphi\right) \leq \operatorname{Disc}\left(\mathcal{A}_{2}, \mathcal{O}, \varphi\right)$.

\section{Constraint Markov Chains, Opacity and Refinement}

\subsection{Constraint Markov Chains and Opacity}

Constraint Markov chains (CMCs) were first introduced in [9] as a specification formalism and further studied in [12. They generalize Interval Markov Chains (IMCs), that were also investigated in [11]13 15] and extended with parameters in [14] with a focus on the consistency problem, i.e., the problem of existence of an implementation satisfying a given specification.

Definition 3.1 (Constraint Markov Chains). A Constraint Markov Chain $(C M C)$ over alphabet $\Sigma$ is a tuple $\mathcal{S}=\left(S, s_{\text {init }}, T, \lambda\right)$ where $S$ is a finite set of states, with $s_{\text {init }} \in S$ the initial state, $T: S \rightarrow 2^{\text {Dist(S) }}$ associates with any state $s \in S$ a set $T(s)$ of distributions over $S$, and $\lambda: S \rightarrow 2^{\Sigma}$ is the labeling function.

The class of CMCs is very general and benefits from nice closure properties as shown in [12. However, without an explicit description of the sets $T(s)$ for $s \in S$, algorithmic questions cannot be solved. For our purpose, we simply consider the subclass of Linear CMCs, where each set $T(s)$ is defined by a conjunction of linear inequalities and the label $\lambda(s)$ is a singleton. In this case, with a slight abuse of notation, we note $\lambda(s)=a$ instead of $\lambda(s)=\{a\}$, as done in Fig. 1(c). By construction, Linear CMCs are closed under conjunction.

More precisely, a linear constraint over $S$ is of the form $\sum_{s \in S} \alpha_{s} x_{s} \bowtie$ $\beta$, with all $\alpha_{s}$ and $\beta$ in $\mathbb{Q}, \bowtie \in\{<, \leq,=, \geq,>\}$, and each $x_{s}$ is a variable for state $s$. A linear probability set on $S$ is a subset of $\operatorname{Dist}(S)$ where the distributions $\mu=(\mu(s))_{s \in S}$ are the solutions of a system of linear constraints over $S$. Remark that the conditions for $\mu$ to be a distribution are also described by linear constraints: $\sum_{s \in S} x_{s}=1$ and, for all $s$, $0 \leq x_{s} \leq 1$; these constraints are implicit in the sequel. Thus a linear probability set is a linear set in $\mathbb{R}^{|S|}$ in the usual sense. It is also a convex polytope. We denote by $\mathbb{L}(S)$ the set of linear probability sets on $S$. 
Definition 3.2 (Linear CMC). A Linear CMC (or shortly $L C M C$ ) is a $C M C \mathcal{S}=\left(S, s_{\text {init }}, T, \lambda\right)$ where for each $s \in S, T(s) \in \mathbb{L}(S)$ and $|\lambda(s)|=1$.

For IMCs, each set $T(s)$ is defined by a family $\left(I\left(s, s^{\prime}\right)\right)_{s^{\prime} \in S}$ of intervals in $[0,1]$ and contains all distributions $\mu$ such that for each $s^{\prime} \in S$, $\mu\left(s^{\prime}\right) \in I\left(s, s^{\prime}\right)$. This can be expressed as a system of linear inequalities by introducing the lower and upper bounds of the intervals. Note that any PTS can be seen as an IMC (hence as an LCMC), where each interval is reduced to a point. For convenience, we keep the interval notation when applicable.

For example, consider the LCMC of Fig. 3, representing a simple system. For graphical depiction, an edge from $s$ to $s^{\prime}$ is labeled by the variable for $x_{s^{\prime}}$ in the system of linear constraints defining $T(s)$; as usual, absence of edge $\left(s, s^{\prime}\right)$ means $x_{s^{\prime}}=0$ is a constraint of $T(s)$. The aim of this system is to achieve Success, but there can be errors (which may be recovered) and failures (which cannot). The probabilities underlying the behavior of the system are not fixed, although a certain number of constraints are known. For example, the probability of a recoverable error is at least twice the one of an unrecoverable one, as expressed by the equation $x_{2} \geq 2 x_{3}$, and when trying to recover from an error, there is a probability of success exceeding the probability of definite failure by $\frac{1}{4}$.

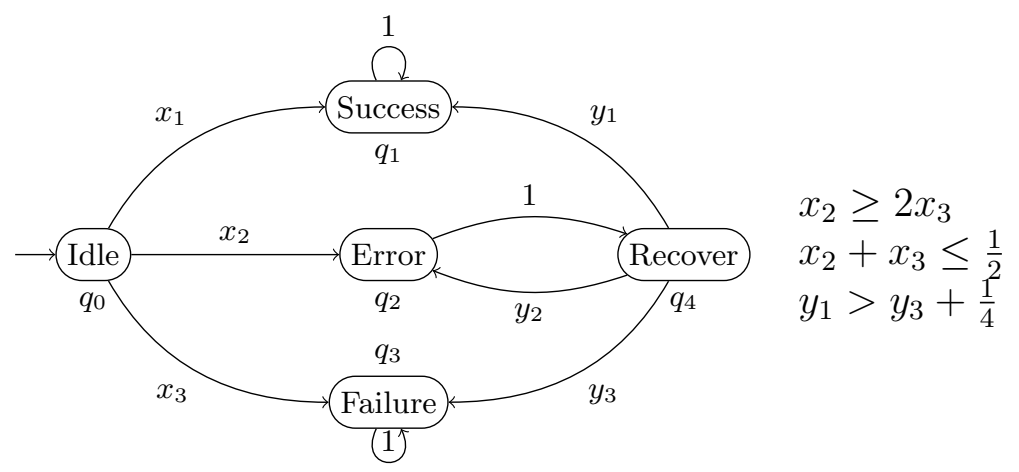

Fig. 3. An LCMC which is not a conjunction of IMCs.

Note that it is not an IMC. Indeed, assume that the constraints on $T\left(q_{0}\right)$ can be expressed by intervals. Any values for $x_{1}, x_{2}$, and $x_{3}$ that satisfy the linear constraints should be in the intervals. In particular, it is the case for the three tuples of values for $\left(x_{1}, x_{2}, x_{3}\right):(1,0,0)$, and $\left(\frac{1}{2}, \frac{1}{2}, 0\right)$, 
$\left(\frac{1}{2}, \frac{1}{3}, \frac{1}{6}\right)$. So it must be the case that $\left[\frac{1}{2}, 1\right] \subseteq I\left(q_{0}, q_{1}\right),\left[0, \frac{1}{2}\right] \subseteq I\left(q_{0}, q_{2}\right)$, and $\left[0, \frac{1}{6}\right] \subseteq I\left(q_{0}, q_{3}\right)$. Hence the distribution defined by $x_{1}=\frac{5}{6}, x_{2}=0$, $x_{3}=\frac{1}{6}$ is within the bounds of the intervals, although it does not satisfy the constraint $x_{2} \geq 2 x_{3}$, which is a contradiction.

In addition, it is neither the result of conjunction of several IMCs, as those only yield constraints where coefficients are positive, hence constraint $x_{2} \geq 2 x_{3}$ (which is actually $x_{2}-2 x_{3} \geq 0$ ) cannot be expressed.

Several semantics can be given to CMCs with respect to the set of PTSs they specify. The simplest one corresponds to first choosing for each state $s$ a distribution belonging to $T(s)$, thus producing a PTS (the implementation), with the same structure as the CMC (the specification). This was done for IMCs in [11,13] and called the Uncertain Markov Chain semantics. A richer semantics consists in introducing a scheduler, choosing the distribution at each step to obtain an implementation, as in a Markov Decision Process (MDP). This was also defined in [11] for IMCs and called IMDP for Interval Markov Decision Process. Finally, the most general semantics corresponds to the satisfaction relation from [912, restricted in 12 to finite state processes. This relation can be obtained from the refinement defined below in Section 3.2 .

We consider here the MDP semantics. A run of $\mathcal{S}$ starting from a state $s$ is a sequence $s \stackrel{\mu_{1}}{\longrightarrow} s_{1} \stackrel{\mu_{2}}{\longrightarrow} \ldots$ where $s_{i} \in S$ and each $\mu_{i}$ is a distribution over $S$ such that $\forall s \in S, \mu_{i} \in T\left(s_{i-1}\right)$. As before, we denote by $\operatorname{Runs}_{s}(\mathcal{S})$ the set of runs starting from $s$, we set $\operatorname{Runs}(\mathcal{S})=\operatorname{Runs}_{\text {sinit }}(\mathcal{S}), \operatorname{FRuns}(\mathcal{S})$ is the set of finite runs of $\mathcal{S}$ starting from $s_{\text {init }}$, and for a run $\rho=s \stackrel{\mu_{1}}{\longrightarrow}$ $s_{1} \stackrel{\mu_{2}}{\longrightarrow} \ldots s_{n-1} \stackrel{\mu_{n}}{\longrightarrow} s_{n}$ in FRuns $(\mathcal{S})$ we $\operatorname{define} \operatorname{lst}(\rho)=s_{n}$.

To associate a probability measure with the runs, it is necessary to resolve the non determinism by a scheduler that chooses a distribution at each step. More precisely:

Definition 3.3 (Scheduler). A scheduler A for a CMC specification $\mathcal{S}=\left(S, s_{\text {init }}, T, \lambda\right)$, is a mapping $A: F R u n s(\mathcal{S}) \rightarrow \operatorname{Dist}(S)$ such that for each run $\rho$ with $s=\operatorname{lst}(\rho), A(\rho) \in T(s)$.

We denote by $\operatorname{Sched}(\mathcal{S})$ the set of schedulers for $\mathcal{S}$. Like for Markov Decision Processes, scheduling $\mathcal{S}$ with $A$ produces a PTS denoted by $\mathcal{S}(A)$, where states are finite runs of $\mathcal{S}$ and the labelings must be consistent: $\mathcal{S}(A)=\left\langle Q, q_{\text {init }}, \Delta, L\right\rangle$ with $Q \subseteq F R u n s(\mathcal{S})$, the initial state is $q_{\text {init }}=s_{\text {init }}$, the run containing only the initial state of $\mathcal{S}$, and for $\rho \in Q$, $L(\rho) \in \lambda(\operatorname{lst}(\rho))$ and $\Delta(\rho)\left(\rho^{\prime}\right)=A(\rho)\left(s^{\prime}\right)$ for $\rho^{\prime}=\rho \stackrel{A(\rho)}{\longrightarrow} s^{\prime}$. We note:

$$
\underline{\operatorname{sat}}(\mathcal{S})=\{\mathcal{S}(A) \mid A \in \operatorname{Sched}(\mathcal{S})\} .
$$


Note that the Uncertain Markov Chains semantics corresponds to the particular case of memoryless schedulers.

We now lift the notion of disclosure to the set of scheduled implementations of a specification $\mathcal{S}$ by:

$$
\operatorname{Disc}(\mathcal{S}, \mathcal{O}, \varphi)=\sup _{A \in \operatorname{Sched}(\mathcal{S})} \operatorname{Disc}(\mathcal{S}(A), \mathcal{O}, \varphi) .
$$

This measure differs from the similar one in [7] for Markov Decision Processes. The notion presented here is finer since the set of runs measured by the disclosure depends on the scheduled implementation. In [7], the set of runs of the disclosure is defined on the (unscheduled) MDP, and its probability is optimized afterwards. This would not be well-defined in CMCs, since two scheduled implementations can have different sets of edges with non-null probability, as explained in Section 4.

\subsection{Refinement}

The notion of strong refinement between probabilistic specifications was introduced in [9] through simulation. A weaker refinement was also proposed in [12]. These notions are adapted to our setting in Definitions 3.4 and 3.5 below.

Definition 3.4 (Strong refinement relation). For two CMC specifications $\mathcal{S}_{1}=\left(S_{1}, s_{1, \text { init }}, T_{1}, \lambda_{1}\right)$ and $\mathcal{S}_{2}=\left(S_{2}, s_{2, \text { init }}, T_{2}, \lambda_{2}\right)$ over alphabet $\Sigma, \mathcal{S}_{1}$ strongly refines $\mathcal{S}_{2}$, written $\mathcal{S}_{1} \preceq_{s} \mathcal{S}_{2}$, if there exists a relation $\mathcal{R} \subseteq S_{1} \times S_{2}$ such that $s_{1, \text { init }} \mathcal{R} s_{2, \text { init }}$ and if $s_{1} \mathcal{R} s_{2}$ then:

(1) $\lambda_{1}\left(s_{1}\right) \subseteq \lambda_{2}\left(s_{2}\right)$,

(2) there exists a function $\delta: S_{1} \rightarrow \mathcal{D}$ ist $\left(S_{2}\right)$ such that for all $\mu \in T_{1}\left(s_{1}\right)$ $\sum_{s_{1}^{\prime} \in S_{1}} \mu\left(s_{1}^{\prime}\right) \cdot \delta\left(s_{1}^{\prime}\right) \in T_{2}\left(s_{2}\right)$,

(3) $s_{1}^{\prime} \mathcal{R} s_{2}^{\prime}$ whenever $\delta\left(s_{1}^{\prime}\right)\left(s_{2}^{\prime}\right)>0$.

For weak refinement, the mapping $\delta$ depends on the chosen distribution $\mu$ (as well as on $s_{1}$ and $s_{2}$ ) instead of being uniform:

Definition 3.5 (Weak refinement relation). For two CMC specifications $\mathcal{S}_{1}=\left(S_{1}, s_{1, \text { init }}, T_{1}, \lambda_{1}\right)$ and $\mathcal{S}_{2}=\left(S_{2}, s_{2, \text { init }}, T_{2}, \lambda_{2}\right)$ over alphabet $\Sigma, \mathcal{S}_{1}$ weakly refines $\mathcal{S}_{2}$, written $\mathcal{S}_{1} \preceq_{w} \mathcal{S}_{2}$, if there exists a relation $\mathcal{R} \subseteq S_{1} \times S_{2}$ such that $s_{1, \text { init }} \mathcal{R} s_{2, \text { init }}$ and if $s_{1} \mathcal{R} s_{2}$ then:

(1) $\lambda_{1}\left(s_{1}\right) \subseteq \lambda_{2}\left(s_{2}\right)$, 


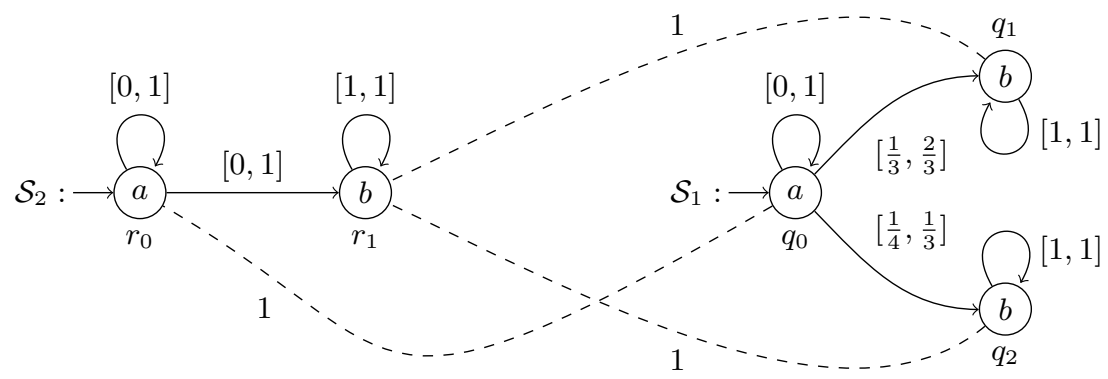

Fig. 4. A refinement of $\mathcal{S}_{2}$ by $\mathcal{S}_{1}$.

(2) for each $\mu \in T_{1}\left(s_{1}\right)$ there exists a function $\delta: S_{1} \rightarrow \operatorname{Dist}\left(S_{2}\right)$ such that $\sum_{s_{1}^{\prime} \in S_{1}} \mu\left(s_{1}^{\prime}\right) \cdot \delta\left(s_{1}^{\prime}\right) \in T_{2}\left(s_{2}\right)$,

(3) $s_{1}^{\prime} \mathcal{R} s_{2}^{\prime}$ whenever $\delta\left(s_{1}^{\prime}\right)\left(s_{2}^{\prime}\right)>0$.

Fig. 4 illustrates the strong refinement relation $\mathcal{R}$ of $\mathcal{S}_{2}$ by $\mathcal{S}_{1}$. For Condition (2) above, we may uniformly use the function: $\delta_{0}\left(q_{i}\right)\left(r_{j}\right)=1$ if $\left(q_{i}, r_{j}\right) \in \mathcal{R}$ and 0 otherwise, represented with dashed lines in Fig. 4 .

Note that there is no weak refinement relation of $\mathcal{S}_{1}$ by $\mathcal{S}_{2}$. Indeed, let $\mu \in T_{2}\left(r_{0}\right)$ defined by $\mu\left(r_{0}\right)=\frac{4}{5}$ and $\mu\left(r_{1}\right)=\frac{1}{5}$. Given any function $\delta$ : $S_{2} \rightarrow \operatorname{Dist}\left(S_{1}\right)$ as in Definition 3.5, it must be the case that $\delta\left(r_{0}\right)\left(q_{1}\right)=0$ since labels do not match. However, $\mu\left(r_{1}\right) \cdot \delta\left(r_{1}\right)\left(q_{1}\right) \leq \mu\left(r_{1}\right)=\frac{1}{5} \notin\left[\frac{1}{3}, \frac{2}{3}\right]$. So $\mu\left(r_{1}\right) \cdot \delta\left(r_{1}\right)$ is not a distribution in $T_{1}\left(q_{0}\right)$, which violates Condition (2).

When a PTS refines a specification, both notions coincide and correspond to the satisfaction relation, which defines the most general semantics of CMCs (from [9]): A PTS $\mathcal{A}=\left(Q, q_{\text {init }}, \Delta, L\right)$ implements a CMC $\mathcal{S}=\left(S, s_{\text {init }}, T, \lambda\right)$, written $\mathcal{A} \vDash \mathcal{S}$ for the associated relation $\vDash \subseteq Q \times P$, if $\mathcal{A}$ refines $\mathcal{S}$, where $\mathcal{A}$ is seen as a CMC with point distributions. Specification $\mathcal{S}$ is said consistent if it admits at least one implementation.

Refinement also applies to two PTSs, and since each probability set reduces to a single distribution, Condition $(2)$ becomes $\left(2^{\prime}\right)$ :

$$
\text { For all } s_{2}^{\prime} \in S_{2}, \sum_{s_{1}^{\prime} \in S_{1}} \Delta_{1}\left(s_{1}\right)\left(s_{1}^{\prime}\right) \cdot \delta\left(s_{1}^{\prime}\right)\left(s_{2}^{\prime}\right)=\Delta_{2}\left(s_{2}\right)\left(s_{2}^{\prime}\right) \text {. }
$$

It is proved in [12] that $\mathcal{S}_{1} \preceq_{s} \mathcal{S}_{2}$ implies $\mathcal{S}_{1} \preceq_{w} \mathcal{S}_{2}$, which in turn implies that for any PTS $\mathcal{A}$, if $\mathcal{A} \vDash \mathcal{S}_{1}$ then $\mathcal{A} \vDash \mathcal{S}_{2}$, all implications being strict. 
Finally, it can be seen that scheduling a CMC specification is a particular case of implementation but not every implementation can be mapped to a scheduler:

Proposition 3.6. Let $\mathcal{S}$ be a $C M C$ specification. For each scheduler $A$ of $\mathcal{S}, \mathcal{S}(A) \vDash \mathcal{S}$.

Proof. The relation $\mathcal{R} \subseteq Q \times S$ is defined by $\mathcal{R}=\{(\rho, s) \mid \operatorname{lst}(\rho)=s\}$. We prove that the relation $\mathcal{R}$ is a refinement relation by defining $\delta_{(\rho, s)}$ over $Q \times S$ as follows:

$$
\delta_{(\rho, s)}\left(\rho^{\prime}, s^{\prime}\right)= \begin{cases}A(\rho)\left(s^{\prime}\right) & \text { if } \rho^{\prime}=\rho \stackrel{A(\rho)}{\longrightarrow} s^{\prime}, \\ 0 & \text { otherwise. }\end{cases}
$$

The first condition results from the definition of the labeling and conditions 2 and 3 come from the fact that the joint distribution $\delta_{(\rho, s)}$ is diagonal in this case.

(2) (a) $\delta\left(\rho^{\prime}, S\right)=A(\rho)\left(s^{\prime}\right)=\Delta(\rho)\left(\rho^{\prime}\right)$ with $\rho^{\prime}=\rho \stackrel{A(\rho)}{\longrightarrow} s^{\prime}$ for all $\rho^{\prime} \in Q$.

(b) $\delta\left(Q, s^{\prime}\right)=A(\rho)\left(s^{\prime}\right) \in T(s)\left(s^{\prime}\right)$ with $s=\operatorname{lst}(\rho)$ for all $s^{\prime} \in S$.

(3) $\rho^{\prime} \mathcal{R} s^{\prime}$ whenever $\delta\left(\rho^{\prime}, s^{\prime}\right)>0$ since $s^{\prime}=\operatorname{lst}\left(\rho^{\prime}\right)$ by definition of $\mathcal{R}$.

Indeed, for any scheduler $A, \mathcal{S}(A)$ is a kind of unfolding of $\mathcal{S}$, which restricts the structure of $\mathcal{S}(A)$ : at each step, the scheduler chooses a valid distribution among successor states. Hence not every implementation can be mapped to a scheduler. Said otherwise, not all implementations can be put in relation with $\mathcal{S}$ through a satisfaction relation where the joint distributions $\delta$ are diagonal.

For example, consider the specification $\mathcal{S}_{0}$ of Fig. 5(a). There is a single possible scheduler for this specification: the one that picks in $q_{0}$ probability $\frac{1}{2}$ to go to either $q_{1}$ or $q_{2}\left(\mathcal{A}_{1}\right.$ in Fig. 5(b)). However, the PTS $\mathcal{A}_{2}$ of Fig. 5(c) is also an implementation of this specification $\left(\mathcal{A}_{2} \models \mathcal{S}_{0}\right)$ where $r_{2}$ is split between $q_{1}$ and $q_{2}$. The corresponding matrix is

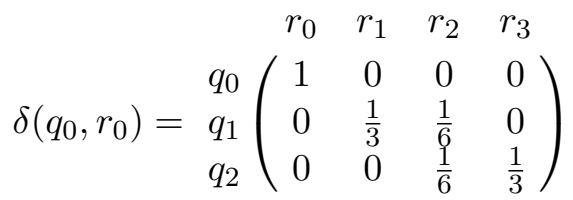

\subsection{Motivating example}

Consider a simple access control database mechanism to a medical database (inspired from [20]) as illustrated in Fig. 6(a), In $\mathcal{S}_{2}$, a user is first requested to input a username $(a)$. If the user name is not on the list of 


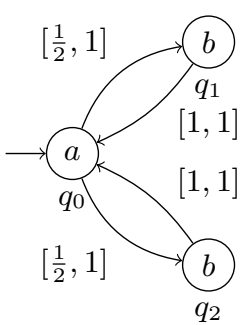

(a) A specification $\mathcal{S}_{0}$

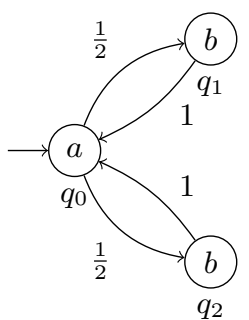

(b) $\mathcal{A}_{1}$, the only scheduling of $\mathcal{S}_{0}$

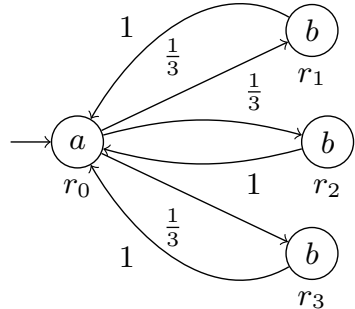

(c) $\mathcal{A}_{2}$, an implementation (not a scheduling) of $\mathcal{S}_{0}$

Fig. 5. A specification with an implementation that is not the result of scheduling.

authorized medical staff $(d)$, the request is rejected $(e)$ and otherwise, the user is requested to input a password $(b)$. If the password is correct, access to the database records is provided $(c)$ and is refused $(e)$, otherwise. The transition probabilities take in accountdepend many factors e.g. the number of legitimate users of the database, the robustness of their respective passwords, or the attacker's knowledge. $\mathcal{S}_{1}$ depicted in Fig. 6(b) refines the password verification process. If the password is correct, it is accepted. Otherwise, a lookup of a black list of common password is performed. If the password is in the list, access is refused, considering it is a malicious attacker and the user is allowed to try again, otherwise. The refinement removes the modalities by restricting intervals (as explained below) and splits the state $q_{1}$ onto $r_{1}$ and $r_{2}$ in $\mathcal{S}_{1}$. In order to express security requirements that any implementation of these specifications should assume, consider the observation function $\mathcal{O}$ defined as $\mathcal{O}(a)=a, \mathcal{O}(c)=c, \mathcal{O}(d)=d, \mathcal{O}(e)=e$ et $\mathcal{O}(b)=\varepsilon$. It reflects the fact that the password input by the user should be kept secret. This could be realized by mean of some cryptographic infrastructure later on along the refinement process but it is not useful to specify this any further here.

It is well known that this kind of mechanism is not robust enough to provide an adequate protection against covert channels allowing an attacker to access any private information from patient medical record whose access should be strictly limited to medical professionals, the legitimate users of the database. As an example, imagine an observer who could discriminate between $a b c^{\omega}$, the execution where a legitimate user is accepted at the first password try and any execution in $a b b^{+} c^{\omega}$ where he 


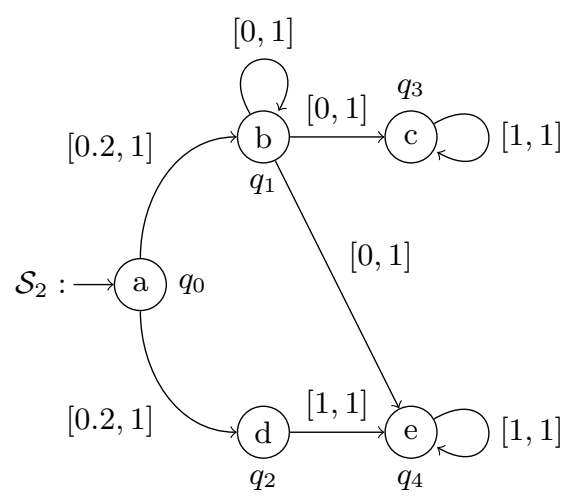

(a) $\mathcal{S}_{2}$

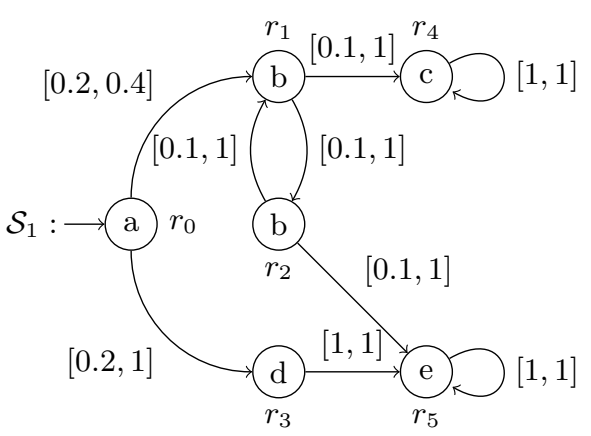

(b) $\mathcal{S}_{1}$

Fig. 6. An access control mechanism to a medical database $\mathcal{S}_{2}$ and its refinement $\mathcal{S}_{1}$.

is only accepted after several tries. This could serve as a channel between this user and an attacker on behalf of whom he is acting for in order to leak any private information of any patient record. Requesting as best opacity as possible of the predicate $\varphi=a b c^{\omega}$, for all implementations of the specification tears down the possibility of such information flow. Let us have a look at the opacity of $\varphi$. First note that $\operatorname{Disc}\left(\mathcal{S}_{2}, \mathcal{O}, \varphi\right)>0$ : since the interval labeling the loop on $q_{1}$ contains 0 , there is a distribution $\mu \in T_{2}\left(q_{1}\right)$ such that $\mu\left(q_{1}\right)=0$. This loop may or may not be scheduled by an attacker and is later called a modal edge for this reason. Hence any implementation $\mathcal{S}_{2}(A)$ of $\mathcal{S}_{2}$ blocking the loop on $q_{1}$ but not the edge $\left(q_{1}, q_{2}\right)$ discloses $\varphi$. Indeed, in this case $\operatorname{Tr}\left(\mathcal{S}_{2}(A)\right)=a b c^{\omega}+a b e^{\omega}+a d e^{\omega}$, $L \backslash \varphi=a b e^{\omega}+a d e^{\omega}, \mathcal{O}(\varphi)=a c^{\omega}$ and $\mathcal{O}(L \backslash \varphi)=a e^{\omega}+a d e^{\omega}$ proving that $\mathcal{O}(\varphi) \nsubseteq \mathcal{O}(L \backslash \varphi)$. Note also that as there is no modal edge in $\mathcal{S}_{1}$, for any scheduler $A, \Delta\left(r_{1}\right)\left(r_{2}\right), \Delta\left(r_{2}\right)\left(r_{1}\right)>0$ where $\Delta$ is the $\mathcal{S}_{1}(A)$ transition function. Hence, $\operatorname{Tr}\left(\mathcal{S}_{1}(A)=a b c^{\omega}+a b(b b)^{*} c^{\omega}+a(b b)^{+} e^{\omega}+a d e^{\omega}\right.$, and $\mathcal{O}(L \backslash \varphi)=a c^{\omega}+a e^{\omega}+a d e^{\omega}$, proving that $\varphi$ is opaque for the infinitely many implementations $\mathcal{S}_{1}(A)$ of $\mathcal{S}_{1}$ that is, $\operatorname{Disc}\left(\mathcal{S}_{1}, \mathcal{O}, \varphi\right)=0$. In Section 4 we give an algorithm to compute the exact disclosure of a specification without modal edges (such as $\mathcal{S}_{1}$ ) and a scheme to approximate the general case (like for $\mathcal{S}_{2}$ ). Finally note that $\operatorname{Disc}\left(\mathcal{S}_{1}, \mathcal{O}, \varphi\right)<$ $\operatorname{Disc}\left(\mathcal{S}_{2}, \mathcal{O}, \varphi\right)$. In Section 5 we prove that amazingly, since in general information flow properties are not preserved under refinement, when $\mathcal{S}_{1}$ refines $\mathcal{S}_{2}$, it is always the case that $\operatorname{Disc}\left(\mathcal{S}_{1}, \mathcal{O}, \varphi\right) \leq \operatorname{Disc}\left(\mathcal{S}_{2}, \mathcal{O}, \varphi\right)$, for any secret $\varphi$ and observation function $\mathcal{O}$. 


\section{Computing the probabilistic disclosure}

\subsection{Modal edges}

When the probability of an edge can be equal to 0 , the corresponding action can be completely blocked by a scheduler. From a modeling point of view, modal edges add a lot of flexibility for refinement. This however means that the range of potential implementations is larger and so it will be harder to obtain meaningful properties. Therefore such edges are desirable in an early modeling phase but less so in the latest refinements. As in [9], we call these edges modal edges, and CMCs that contain such edges are called modal CMCs.

Definition 4.1 (Modal edge). An edge $\left(s, s^{\prime}\right)$ in $C M C \mathcal{S}$ is modal if there exists a scheduler $A$ such that $\Delta(\rho)\left(\rho \stackrel{A(\rho)}{\longrightarrow} s^{\prime}\right)=0$ for any run $\rho$ of $\mathcal{S}(A)$ with $\operatorname{lst}(\rho)=s$.

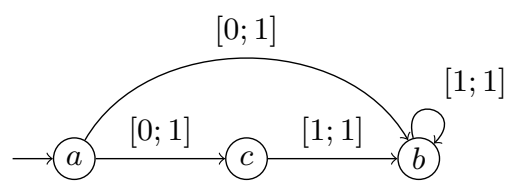

(a) A modal IMC $\mathcal{S}_{\mathrm{m}}$.

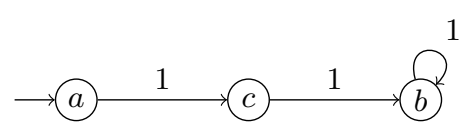

(c) A disclosing implementation of $\mathcal{S}_{\mathrm{m}}$ (but not of $\left.\mathcal{S}_{\mathrm{nm}}\right)$.

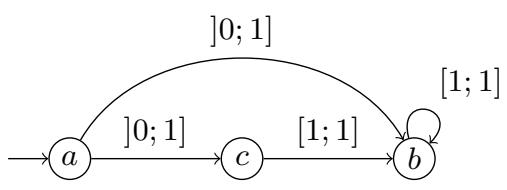

(b) A non-modal IMC $\mathcal{S}_{\text {nm }}$.

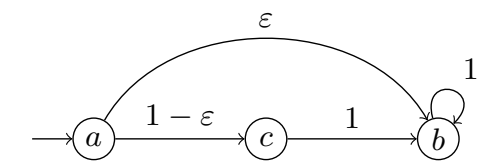

(d) A non-disclosing implementation of $\mathcal{S}_{\mathrm{nm}}\left(\right.$ and $\left.\mathcal{S}_{\mathrm{m}}\right), \varepsilon>0$.

Fig. 7. The influence of modal transitions on disclosure.

In the context of opacity, removing an edge drastically changes the disclosure, since it can remove ambiguities. For example, consider the modal IMC $\mathcal{S}_{\mathrm{m}}$ of Fig. 7(a), where $a$ and $b$ are observed and the secret is the presence of $c$. An implementation of $\mathcal{S}_{\mathrm{m}}$ that blocks the direct edge from $a$ to $b$ (Fig. 7(c) has a disclosure of 1 , since the secret is guaranteed to be part of the only possible run. On the other hand, in the non-modal version of the IMC (Fig. 7(b) , such implementations are banned and only implementations that retain a small probability to avoid $c$ are allowed. In 
these implementations, the disclosure is 0 , since every run is observed as $a b^{\omega}$ and it is possible that $c$ did not occur.

The detection of modal edges is the first step toward computation of the disclosure.

Proposition 4.2. The set of modal edges can be computed in time polynomial in the number of edges.

Proof. The procedure for each edge $\left(s, s^{\prime}\right)$ is as follows. Assume $T(s)$ is defined by the conjunction $\bigwedge_{i=1}^{k} C_{i}$ where each $C_{i}$ is a linear constraint. The edge $\left(s, s^{\prime}\right)$ is modal if and only if $x_{s^{\prime}}=0 \wedge \bigwedge_{i=1}^{k} C_{i}$ admits a solution. This can be checked in polynomial time [21].

\subsection{Computation in non-modal LCMCs}

In the case of non-modal LCMCs, the disclosure can be computed:

Theorem 4.3. Computing the value of disclosure for an $L C M C \mathcal{S}$ without modal edges can be done in 2EXPTIME.

Proof. The proof relies on constructing an (exponentially larger) MDP on which an optimization problem is solved. In the spirit of [22, the construction of the MDP relies on basic feasible solutions (BFSs), otherwise called corner points.

Starting from a DPA $\mathcal{A}_{\varphi}$ for $\varphi$, a DPA $\mathcal{A}_{\mathcal{V}}$ for $\mathcal{V}\left(\mathcal{A}_{K}, \mathcal{O}, \varphi\right)$ can be built, with size exponential in the size of $\mathcal{S}$ and $\mathcal{A}_{\varphi}$ (and with a number $k$ of colors polynomial in the size of $\mathcal{A}$ and $\mathcal{A}_{\varphi}$ ). This construction relies on intersections and complementations of DPA, with a determinization step that brings the exponential blowup [23. Synchronizing the DPA $\mathcal{A}_{\mathcal{V}}$ with the original LCMC yields an LCMC $\mathcal{S}_{\mathcal{V}}$. Finding the optimal scheduler for $\mathcal{S}_{\mathcal{V}}$ to accept yields the optimal value of $\operatorname{Disc}\left(\mathcal{A}_{K}, \mathcal{O}, \varphi\right)$. This optimization is done by translating $\mathcal{S}_{\mathcal{V}}$ into an MDP $\mathcal{M}_{\mathcal{V}}$ as follows.

For each state $s$ of $\mathcal{S}_{\mathcal{V}}$, we compute the set of the BFS of the polytope defined by $T(s)$. Then we build the corresponding state in the MDP $\mathcal{M}_{\mathcal{V}}$ by adding a transition (i.e. a probability distribution) per BFS. As a property of BFSs, any distribution in $T(s)$ in $\mathcal{S}_{\mathcal{V}}$ can be expressed as a barycentric combination of BFSs. Hence a scheduler on $\mathcal{M}_{\mathcal{V}}$ corresponds exactly to a scheduler on $\mathcal{S}_{\mathcal{V}}$. As a result maximizing the probability of $\mathcal{V}\left(\mathcal{A}_{K}, \mathcal{O}, \varphi\right)$ in $\mathcal{M}_{\mathcal{V}}$ is exactly the same as computing said optimum in $\mathcal{S}_{\mathcal{V}}$. This yields a memoryless scheduler, which in turn can be translated into a finite memory scheduler in $\mathcal{S}$. 
Note that this construction annihilates the difference between strict and large inequalities. This is of no consequence on the computation of the value of the disclosure. Indeed, if the optimal value is reached on a facet of the polytope defined through a strict inequality, one can build a sequence of ever closer schedulers converging in the limit to the optimal value. In the case where the strict constraint is of the form $x_{s}>0$, i.e. that the edge should disappear in the limit, this actually does not introduce a modal edge in the sense that the set $\mathcal{V}\left(\mathcal{A}_{K}, \mathcal{O}, \varphi\right)$ is not changed.

The number of BFSs of a system of rank $r$ in dimension $n$ is bounded by $\left(\begin{array}{l}n \\ r\end{array}\right)$ (the number of subsets of cardinality $r$ in a set of $n$ elements), hence for each state there is an exponential number of BFSs to consider. As a result the overall complexity of the procedure is in 2EXPTIME.

An example of the transformation described in the above proof is illustrated for state $q_{0}$ of the LCMC in Fig. 3. The BFSs are depicted in Fig. 8, all possible distributions are points in the red triangle with corners $\mu_{1}, \mu_{2}$, and $\mu_{3}$ being the three BFSs. Note that these distributions only consider values for $x_{1}, x_{2}, x_{3}$, since all other values are null. The transformation of the LCMC (for state $q_{0}$ ) into an MDP is illustrated in Fig. 9 .

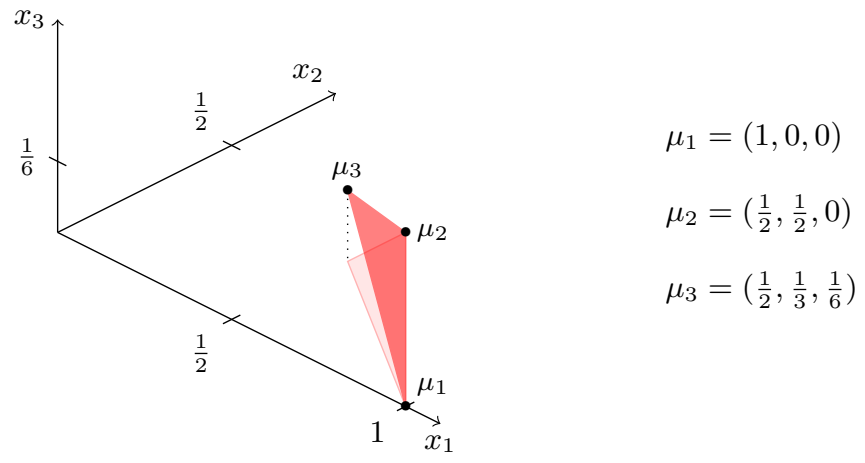

Fig. 8. Basic Feasible Solutions for state $q_{0}$.

\subsection{Towards the general case}

When a scheduler is faced with the choice to include or exclude a modal edge, it can produce several versions of PTSs, say $\mathcal{A}_{1}$ and $\mathcal{A}_{2}$, with $\operatorname{Tr}\left(\mathcal{A}_{1}\right) \neq \operatorname{Tr}\left(\mathcal{A}_{2}\right)$, hence $\mathcal{V}\left(\mathcal{A}_{1}, \mathcal{O}, \varphi\right) \neq \mathcal{V}\left(\mathcal{A}_{2}, \mathcal{O}, \varphi\right)$. In addition, these 

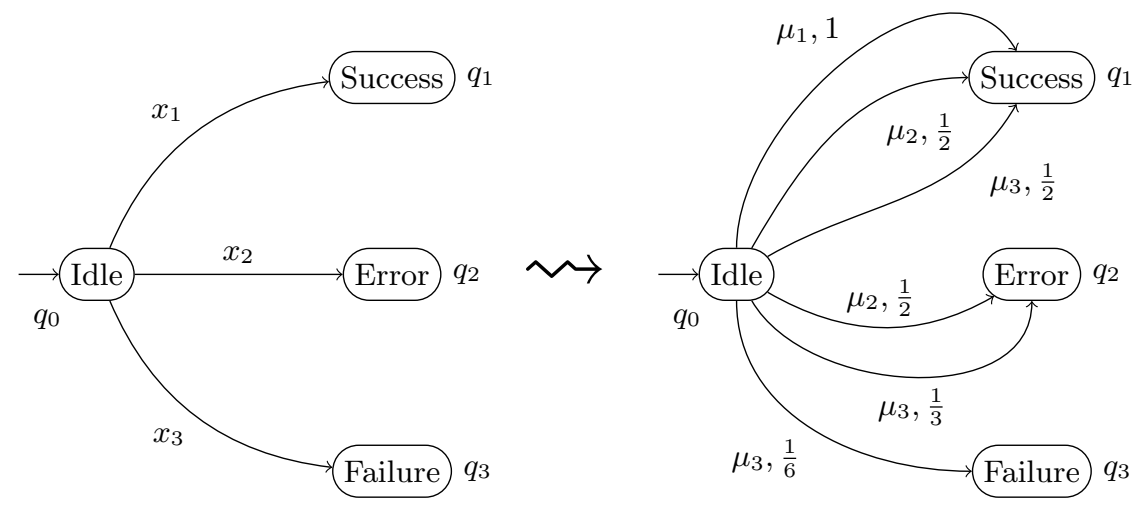

Fig. 9. Transforming an LCMC into an MDP using BFSs.

choices may be history dependent, as in the example of Fig. 10, with $\varphi=a \Sigma^{\omega}$ and only letters $c$ and $d$ being observed. Intuitively, a way for the scheduler to always disclose the presence of an initial $a$ is to always follow an $a$ by the same letter, say a $c$. However, this choice must be made after the first letter has been seen. Moreover, leaving the possibility of a run $a d \cdots$ to occur means that run $a c \cdots$ does not disclose $\varphi$. As a result, the scheduler should also take into account $\varphi$ and the observation function before committing to a choice with respect to modal edges.

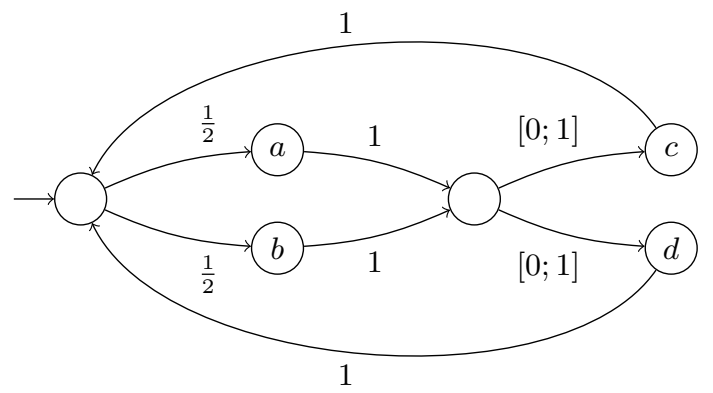

Fig. 10. IMC where the choice on modal edge requires history.

So far, the general case of modal LCMCs remains open. However, we now propose an approximation scheme using finite memory schedulers. 
In the case of modal LCMCs, disclosure can be approximated by computing only what can be disclosed by an adversary with bounded memory. Increasing the allotted memory provides a better approximation of the disclosure, although there is no guarantee that the disclosure can be achieved with finite memory. A finite memory adversary can be defined as follows:

Definition 4.4 (n-memory scheduler). Let $[n]$ denote the set $\{1,2, \ldots, n\}$. An n-memory scheduler $A$ for an $L C M C$ specification $\mathcal{S}=\left(S, s_{\text {init }}, T, \lambda\right)$, is a tuple $A=\left([n], i_{\text {init }}, \theta, \gamma\right)$ where $[n]$ is the set of modes, $i_{\text {init }}$ is the starting mode, $\theta:[n] \times S \rightarrow[n]$ is a mode transition function and $\gamma:[n] \times S \rightarrow \mathcal{D}$ ist $(S)$, is the choice function with $\gamma(i, s) \in T(s)$ for all $i \in[n]$ and all $s \in S$.

Scheduling $\mathcal{S}$ with $A$ produces a PTS $\mathcal{S}(A)$ where the set of states is $[n] \times S$, the initial state is $\left(i_{\text {init }}, s_{\text {init }}\right)$, for $(i, s) \in[n] \times S, L(i, s) \in \lambda(s)$ and $\theta(i, s)\left(i^{\prime}, s^{\prime}\right)=\gamma(i, s)\left(s^{\prime}\right)$ for $i^{\prime}=\theta(i, s)$. We denote by $\operatorname{Sched}_{n}(\mathcal{S})$ the set of $n$-memory schedulers for $\mathcal{S}$, with $\underline{\operatorname{sat}}_{n}(\mathcal{S})=\left\{\mathcal{S}(A) \mid A \in \operatorname{Sched}_{n}(\mathcal{S})\right\}$ and

$$
\operatorname{Disc}_{n}(\mathcal{S}, \mathcal{O}, \varphi)=\sup _{A \in \operatorname{Sched}_{n}(\mathcal{S})} \operatorname{Disc}(\mathcal{S}(A), \mathcal{O}, \varphi) .
$$

Memoryless schedulers are those in $\operatorname{Sched}_{1}(\mathcal{S})$ and the set of finite memory schedulers is $\bigcup_{n} \operatorname{Sched}_{n}(\mathcal{S})[24$.

The computation of the disclosure of an LCMC $\mathcal{S}$ under bounded memory adversaries relies on:

- the computations of the set of modal edges of $\mathcal{S}$ (Proposition 4.2);

- the value of disclosure for LCMCs without modal edges (Theorem 4.3);

- the unwinding of $\mathcal{S}$ with respect to a memory bound and the removal of modal edges (described below).

The unwinding construction. Given $\mathcal{S}=\left(S, s_{\text {init }}, T, \lambda\right)$ and a finite transition system $\mathcal{A}_{n, \theta}=\left([n], i_{\text {init }}, \theta\right)$, we construct the LCMC $\mathcal{A}_{n, \theta} \times \mathcal{S}=$ $\left([n] \times S,\left(i_{\text {init }}, s_{\text {init }}\right), \hat{T}, \hat{\lambda}\right)$ s.t.

- $\hat{\mu} \in \hat{T}(i, s)$ iff $\exists \mu \in T(s)$ such that for $i^{\prime} \in[n]$,

$$
\hat{\mu}\left(i^{\prime}, s^{\prime}\right)=\left\{\begin{array}{l}
\mu\left(s^{\prime}\right) \text { if } i^{\prime}=\theta(i, s) \\
0 \text { otherwise }
\end{array}\right.
$$

$-\hat{\lambda}(i, s)=\lambda(s)$

The unwinding of $\mathcal{S}$ by an $n$-state automaton $\mathcal{A}$ produces an LCMC $\mathcal{S}_{\mathcal{A}}$ formed with $n$ copies of $\mathcal{S}$ (one for each state of $\mathcal{A}$ ), communicating with 
each other according to the mode transition function of $\mathcal{A}$. Memoryless schedulers on $\mathcal{S}_{\mathcal{A}}$ then correspond to $n$-memory schedulers on $\mathcal{S}$. We note $\operatorname{Sched}_{\mathcal{A}_{n, \theta}}(\mathcal{S})$ the set of schedulers for $\mathcal{S}$ of the form $A=\left(\mathcal{A}_{n, \theta}, \gamma\right)$ for $\gamma:[n] \times S \rightarrow \mathcal{D} \operatorname{ist}(S)$ where for $s \in S$ and for all $i \in[n], \gamma(i, s) \in T(s)$. Hence, any implementation of $\underline{s a t}_{n}(\mathcal{S})$ can be obtained by a memoryless scheduler on some $\mathcal{S}_{\mathcal{A}}$. Remark that there is only a finite number of such automata. This construction entails:

Lemma 4.5. For any $L C M C \mathcal{S}$,

$$
\underline{s a t}_{1}\left(\mathcal{A}_{n, \theta} \times \mathcal{S}\right)=\left\{\mathcal{S}(A) \mid A=\left(\mathcal{A}_{n, \theta}, \gamma\right) \text { for some } \gamma\right\} .
$$

The modal edge removing construction. Given $\mathcal{S}=\left(S, s_{\text {init }}, T, \lambda\right)$, an LCMC, let $\mathcal{M}(\mathcal{S})$ be the set of modal edges of $\mathcal{S}$. For a subset $\xi \subseteq \mathcal{M}(\mathcal{S})$, we define $\mathcal{S} \backslash \xi$ as the modal edge free LCMC obtained from $\mathcal{S}$ by removing edges in $\xi$ and "unmodalizing" modal edges of $\mathcal{S}$ not in $\xi$ : this means that if $\left(s, s^{\prime}\right) \in \xi$, we add the constraint $x_{s^{\prime}}=0$ to $T(s)$ and if $\left(s, s^{\prime}\right) \notin \xi$, we add the constraint $x_{s^{\prime}}>0$ to $T(s)$. Note that removing an arbitrary subset $\xi$ of $\mathcal{M}(\mathcal{S})$ may result in an inconsistent specification. These will be ignored by our algorithm (recall that consistency checks are polynomial for LCMCs [12]).

The algorithm. Algorithm 1 enumerates all possible finite transition systems with $n$ states and unwinds $\mathcal{S}$ over them. For each such unwinding, the algorithm explores the set of memoryless schedulers. This exploration is done by first selecting the set of modal edges to remove and for each set, compute the maximal disclosure using the procedure of Theorem 4.3 . Since this procedure may use arbitrary schedulers, the output of the algorithm is an overapproximation of $\operatorname{Disc}_{n}(\mathcal{S}, \mathcal{O}, \varphi)$, the disclosure of $\mathcal{S}$ restricted to $n$-memory schedulers. On the other hand, it is an underapproximation of the actual disclosure.

Correctness. The partial correctness of Algorithm 1 relies on Lemma 4.5 above as well as the following observation, indicating that the choice of some set of modal edges to remove exactly corresponds to the scheduler choice:

Lemma 4.6. For any $L C M C \mathcal{S}$ and for any memoryless scheduler $A=$ $(\mathcal{A}, \gamma)$ of $\mathcal{S}$, there exists a maximal subset $\xi$ of $\mathcal{M}(\mathcal{S})$ such that for any $\left(s, s^{\prime}\right) \in \mathcal{M}(\mathcal{S}), \gamma(1, s)\left(s^{\prime}\right)=0$ iff $\left(s, s^{\prime}\right) \in \xi$.

Proof. Since the scheduler is memoryless, the choice of scheduling a modal edge to 0 must be uniformly taken along any run, hence this edge never 


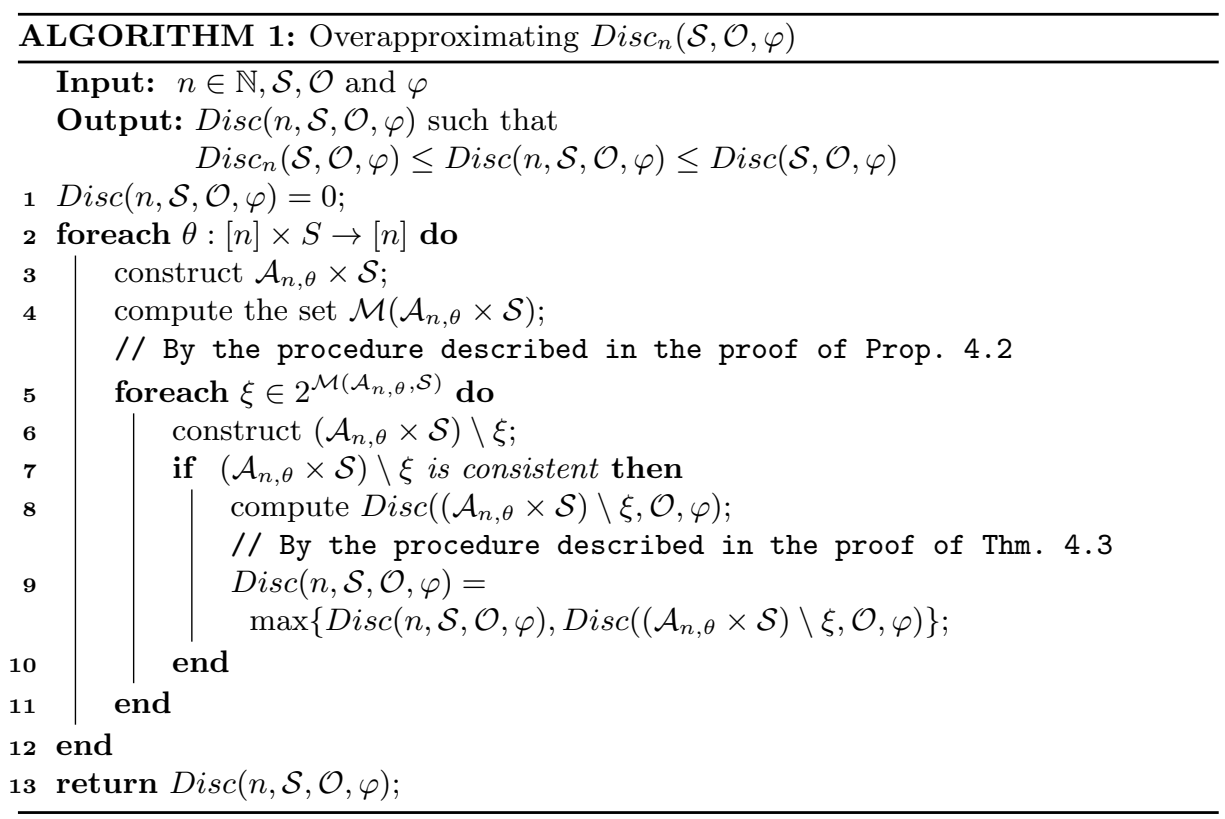

appears in the PTS scheduled by $A$. This is equivalent to remove this edge from $\mathcal{S}$ so the set $\xi$ is the collection of all such edges.

Theorem 4.7 (Correctness of Algorithm 1). Given a an LCMC S , an observation $\mathcal{O}$ and a secret $\varphi$ for $\mathcal{S}$, the disclosure of $\varphi$ in $\mathcal{S}$ for $\mathcal{O}$ by a polynomial size adversary (in the size of $\mathcal{S}$ ) can be over-approximated in $2 E X P T I M E$.

Proof. We prove that the output of Algorithm 1

$$
\operatorname{Disc}(n, \mathcal{S}, \mathcal{O}, \varphi)=\max _{\theta} \max _{\xi} \operatorname{Disc}\left(\mathcal{A}_{n, \theta} \times \mathcal{S} \backslash \xi, \mathcal{O}, \varphi\right)
$$

satisfies the post-condition $\operatorname{Disc}_{n}(\mathcal{S}, \mathcal{O}, \varphi) \leq \operatorname{Disc}(n, \mathcal{S}, \mathcal{O}, \varphi) \leq \operatorname{Disc}(\mathcal{S}, \mathcal{O}, \varphi)$. For the first inequality, let $A=(\mathcal{A}, \gamma)$ be an $n$-memory scheduler for $\mathcal{S}$ and let $\mathcal{S}_{\mathcal{A}}$ be the corresponding unwinding. This unwinding is computed in the loop of Algorithm 1 at line 3 . By Lemma 4.5 the $n$-memory schedulers on $\mathcal{S}$ are exactly the memoryless schedulers on $\mathcal{S}_{\mathcal{A}}$. Hence the choice function $\gamma$ is memoryless on $\mathcal{A} \times \mathcal{S}$. Therefore Lemma 4.6 provides a set $\xi$ of modal edges to be removed, such that $\mathcal{S}_{\mathcal{A}}$ and $\mathcal{S}_{\mathcal{A}} \backslash \xi$ coincide when scheduled by $\gamma$. The removal of this set $\xi$ is performed in the inner loop at line 6. Hence the disclosure of $\mathcal{S}(A)$ is less than or equal to $\operatorname{Disc}\left(\mathcal{S}_{\mathcal{A}} \backslash \xi, \mathcal{O}, \varphi\right)$ 
computed at line 8 (equality is not ensured since the computation of Theorem 4.3 does not restrict memory). This value is itself smaller than the output of the algorithm, hence $\operatorname{Disc}_{n}(\mathcal{S}, \mathcal{O}, \varphi) \leq \operatorname{Disc}(n, \mathcal{S}, \mathcal{O}, \varphi)$.

The second inequality results from the fact that all values computed by Algorithm 1 are of the form $\operatorname{Disc}\left(\mathcal{S}_{\mathcal{A}} \backslash \xi, \mathcal{O}, \varphi\right)$. Since the maximum is obtained on a subset of schedulers, $\operatorname{Disc}(n, \mathcal{S}, \mathcal{O}, \varphi) \leq \operatorname{Disc}(\mathcal{S}, \mathcal{O}, \varphi)$ holds.

This algorithm makes $2^{O(n)}$ calls to the 2EXPTIME procedure of Theorem 4.3. Thus the complexity of Algorithm1 1 remains in 2EXPTIME as long as $n$ is polynomial in the size of $\mathcal{S}$.

\section{Monotonicity of disclosure}

This section is devoted to the proof of the following result, establishing monotonicity with respect to weak refinement for the disclosure over scheduled implementations:

Theorem 5.1. Let $\mathcal{S}_{1}$ and $\mathcal{S}_{2}$ be LCMC specifications such that $\mathcal{S}_{1} \preceq_{w}$ $\mathcal{S}_{2}$. Then $\operatorname{Disc}\left(\mathcal{S}_{1}, \mathcal{O}, \varphi\right) \leq \operatorname{Disc}\left(\mathcal{S}_{2}, \mathcal{O}, \varphi\right)$.

Since scheduling is a restrictive way to derive implementations from a specification, it is not the case in general that $\underline{\operatorname{sat}}\left(\mathcal{S}_{1}\right) \subseteq \underline{\operatorname{sat}}\left(\mathcal{S}_{2}\right)$ : although any scheduling $\mathcal{S}_{1}\left(A_{1}\right)$ of $\mathcal{S}_{1}$ with $A_{1}$ is an implementation of $\mathcal{S}_{2}$, this implementation may not be a scheduling.

Instead, the proof builds a scheduler $A_{2}$ for $\mathcal{S}_{2}$, producing an implementation $\mathcal{S}_{2}\left(A_{2}\right)$ that is refined by $\mathcal{S}_{1}\left(A_{1}\right)$ (Theorem 5.2, illustrated in Fig. 11). Then, this refinement is shown to ensure that the probabilities of (cones of) finite words coincide (Propositions 5.3 and 5.4). The disclosure set being a measurable event, coincidence of probabilities on cones ensures coincidence of probabilities for the disclosure.

Notations. Given two specifications $\mathcal{S}_{1}$ and $\mathcal{S}_{2}$ such that $\mathcal{S}_{1}$ weakly refines $\mathcal{S}_{2}$ through relation $\mathcal{R}$, we define the relation $\sim$ on $\operatorname{FRuns}\left(\mathcal{S}_{1}\right) \times$ $\operatorname{FRuns}\left(\mathcal{S}_{2}\right)$ by: $\rho_{1} \sim \rho_{2}$ if $\left|\rho_{1}\right|=\left|\rho_{2}\right|$ and at any intermediate step $i$, the corresponding states satisfy $s_{1, i} \mathcal{R} s_{2, i}$.

For $\rho_{2} \in \operatorname{FRuns}\left(\mathcal{S}_{2}\right)$, we set $\operatorname{sim}\left(\rho_{2}\right)=\left\{\rho_{1} \in \operatorname{FRuns}\left(\mathcal{S}_{1}\right) \mid \rho_{1} \sim \rho_{2}\right\}$. We now define a measure $\mu_{\rho_{2}}$ over $\operatorname{sim}\left(\rho_{2}\right)$ by $\mu_{\rho_{2}}\left(\rho_{1}\right)=\frac{\mathbf{P}_{\mathcal{A}_{1}}\left(\rho_{1}\right)}{\mathbf{P}_{\mathcal{A}_{1}}\left(\operatorname{sim}\left(\rho_{2}\right)\right)}$ (where the probability of finite run $\rho$ is abusively written instead of the probability of its cone $C_{\rho}$ ).

We first show how to build a scheduler $A_{2}$ for $\mathcal{S}_{2}$ such that $\mathcal{S}_{1}\left(A_{1}\right)$ refines $\mathcal{S}_{2}\left(A_{2}\right)$. Recall from Section 3.2 that for PTSs, weak and strong re- 


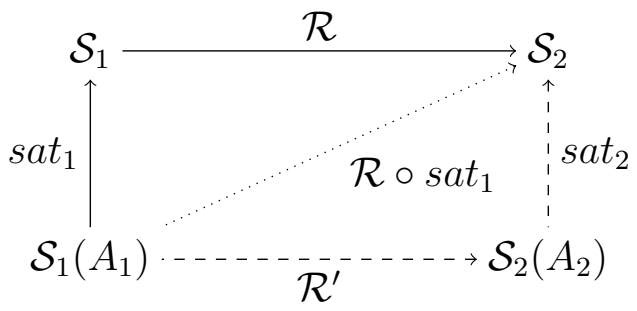

Fig. 11. Result of Theorem 5.2, Relation $\mathcal{R} \circ s_{1} t_{1}$ always exists but might not be a scheduling.

finement coincide and are thus simply called refinement in the remainder of this section.

Theorem 5.2. Let $\mathcal{S}_{1}$ and $\mathcal{S}_{2}$ be LCMC specifications such that $\mathcal{S}_{1}$ weakly refines $\mathcal{S}_{2}$. Then for any $A_{1} \in \operatorname{Sched}\left(\mathcal{S}_{1}\right)$ there exists $A_{2} \in \operatorname{Sched}\left(\mathcal{S}_{2}\right)$ such that $\mathcal{S}_{1}\left(A_{1}\right)$ refines $\mathcal{S}_{2}\left(A_{2}\right)$.

Proof. Let $\mathcal{S}_{1}=\left(S_{1}, s_{\text {init }, 1}, T_{1}, \lambda_{1}\right)$ and $\mathcal{S}_{2}=\left(S_{2}, s_{\text {init }, 2}, T_{2}, \lambda_{2}\right)$ be LCMCs such that $\mathcal{S}_{1}$ refines $\mathcal{S}_{2}$ with $\mathcal{R}$. Let $s a t_{1}$ be the satisfaction relation related to $A_{1}$ and $\mathcal{A}_{1}=\mathcal{S}_{1}\left(A_{1}\right)=\left(Q_{1}, q_{\text {init }, 1}, \Delta_{1}, L_{1}\right)$. Then we show that there exists $A_{2} \in \operatorname{Sched}\left(\mathcal{S}_{2}\right)$ and a refinement relation $\mathcal{R}^{\prime}$ such that $\mathcal{R} \circ \operatorname{sat}_{1}=$ $s t_{2} \circ \mathcal{R}^{\prime}$ where $s_{2} t_{2}$ is the satisfaction relation related to $A_{2}$.

Let $\rho_{2} \in F \operatorname{Runs}\left(\mathcal{S}_{2}\right)$ with $\operatorname{lst}\left(\rho_{2}\right)=s_{2}$. Then, for any $\rho_{1} \in \operatorname{sim}\left(\rho_{2}\right)$, $A_{1}\left(\rho_{1}\right) \in T_{1}\left(\operatorname{lst}\left(\rho_{1}\right)\right)$ and $\operatorname{lst}\left(\rho_{1}\right) \mathcal{R} s_{2}$. Since $\mathcal{S}_{1}$ weakly refines $\mathcal{S}_{2}$, there exists $\delta_{\rho_{1}}: S_{1} \rightarrow \operatorname{Dist}\left(S_{2}\right)$ such that $\sum_{s_{1}^{\prime} \in S_{1}} A_{1}\left(\rho_{1}\right)\left(s_{1}^{\prime}\right) \delta_{\rho_{1}}\left(s_{1}^{\prime}\right) \in T_{2}\left(s_{2}\right)$.

We define $A_{2}$ on FRuns $\left(\mathcal{S}_{2}\right)$ by:

$$
A_{2}\left(\rho_{2}\right)=\sum_{\rho_{1} \in \operatorname{sim}\left(\rho_{2}\right)} \mu_{\rho_{2}}\left(\rho_{1}\right) \sum_{s_{1}^{\prime} \in S_{1}} A_{1}\left(\rho_{1}\right)\left(s_{1}^{\prime}\right) \cdot \delta_{\rho_{1}}\left(s_{1}^{\prime}\right) .
$$

From the definition of $\mu_{\rho_{2}}$ and the convexity of $T_{2}\left(s_{2}\right)$, we can conclude that $A_{2}\left(\rho_{2}\right)$ also belongs to $T_{2}\left(s_{2}\right)$, hence $A_{2}$ is a scheduler of $\mathcal{S}_{2}$.

Writing now $\mathcal{A}_{2}=\mathcal{S}_{2}\left(A_{2}\right)=\left(Q_{2}, q_{\text {init, } 2}, \Delta_{2}, L_{2}\right)$, the relation $\mathcal{R}^{\prime}$ can be defined as $\sim$ (relating runs that are similar "step by step", as defined above). To see that the conditions are satisfied, let $\rho_{1}$ and $\rho_{2}$ be runs in $Q_{1}$ and $Q_{2}$ respectively. Then the mapping $\delta^{\prime}: Q_{1} \rightarrow \operatorname{Dist}\left(Q_{2}\right)$ is obtained by:

$$
\delta^{\prime}\left(\rho_{1}\right)\left(\rho_{2}\right)= \begin{cases}\mu_{\rho_{2}}\left(\rho_{1}\right) \delta_{\rho_{1}}\left(\operatorname{lst}\left(\rho_{1}\right)\right)\left(\operatorname{lst}\left(\rho_{2}\right)\right) & \text { if } \rho_{1} \sim \rho_{2}, \\ 0 & \text { otherwise. }\end{cases}
$$

Since $\mathcal{A}_{1}$ and $\mathcal{A}_{2}$ are PTSs, we just need to show that Equation 3.2 holds. Writing $\rho_{2}^{\prime}=\rho_{2} \stackrel{A_{2}\left(\rho_{2}\right)}{\longrightarrow} s_{2}^{\prime}$, we have: 


$$
\begin{aligned}
\Delta_{2}\left(\rho_{2}\right)\left(\rho_{2}^{\prime}\right) & =A_{2}\left(\rho_{2}\right)\left(s_{2}^{\prime}\right) \\
& =\sum_{\rho_{1} \in \operatorname{sim}\left(\rho_{2}\right)} \mu_{\rho_{2}}\left(\rho_{1}\right) \sum_{s_{1}^{\prime} \in S_{1}} A_{1}\left(\rho_{1}\right)\left(s_{1}^{\prime}\right) \cdot \delta_{\rho_{1}}\left(s_{1}^{\prime}\right)\left(s_{2}^{\prime}\right) \\
& =\sum_{\rho_{1} \in \operatorname{sim}\left(\rho_{2}\right)} \sum_{s_{1}^{\prime} \in S_{1}} A_{1}\left(\rho_{1}\right)\left(s_{1}^{\prime}\right) \cdot \delta^{\prime}\left(\rho_{1} \stackrel{A\left(\rho_{1}\right)}{\longrightarrow} s_{1}^{\prime}\right)\left(\rho_{2}^{\prime}\right) \\
& =\sum_{\rho_{1}^{\prime} \in Q_{1}} A_{1}\left(\rho_{1}\right)\left(s_{1}^{\prime}\right) \cdot \delta^{\prime}\left(\rho_{1}^{\prime}\right)\left(\rho_{2}^{\prime}\right)
\end{aligned}
$$

by defining $\rho_{1}^{\prime}=\rho_{1} \stackrel{A_{1}\left(\rho_{1}\right)}{\longrightarrow} s_{1}^{\prime}$ for each $\rho_{1}$ and remarking that $\delta^{\prime}=0$ if its arguments are not similar runs. Hence:

$$
\Delta_{2}\left(\rho_{2}\right)\left(\rho_{2}^{\prime}\right)=\sum_{\rho_{1}^{\prime} \in Q_{1}} \Delta_{1}\left(\rho_{1}\right)\left(\rho_{1}^{\prime}\right) \cdot \delta^{\prime}\left(\rho_{1}^{\prime}\right)\left(\rho_{2}^{\prime}\right)
$$

Now we show that refinement between two PTSs is sufficient to compare their disclosure. Namely, we show that the probabilities of cones of words are equal in both systems. Note that although this property is well known to hold for paths, it needs to be lifted to words in order to compare disclosure.

We start by considering the sets of traces globally; although it is folklore that refinement implies trace inclusion, we provide a proof for completeness sake.

Proposition 5.3. Let $\mathcal{A}_{1}$ and $\mathcal{A}_{2}$ be PTSs such that $\mathcal{A}_{1}$ refines $\mathcal{A}_{2}$. Then $\operatorname{Tr}\left(\mathcal{A}_{1}\right)=\operatorname{Tr}\left(\mathcal{A}_{2}\right)$.

Proof. We prove the proposition by induction on a strengthened statement. Namely, we claim that for every finite run in $\mathcal{A}_{1}$ there exists a similar run in $\mathcal{A}_{2}$. Since an infinite run is the limit of the sequence of its finite prefixes, this claim is sufficient to prove the proposition. Assume by induction that the proposition holds for every word of length $n$. Let $w \in F \operatorname{Tr}\left(\mathcal{A}_{1}\right)$ of length $n+1$. We write $w=w_{0} a$ for some $a \in \Sigma$. Consider a run of $\mathcal{A}_{1}$ that produces $w$. It is of the form $\rho_{0} s_{1}^{\prime}$ where $\lambda\left(s_{1}^{\prime}\right)=a$; let $s_{1}=\operatorname{lst}\left(\rho_{0}\right)$. Let $\rho_{0}^{\prime}$ be a run in $\mathcal{A}_{2}$, similar to $\rho_{0}$, and $s_{2}=\operatorname{lst}\left(\rho_{0}^{\prime}\right)$. By definition of refinement, there exists a function $\delta$ such that for any state $s_{2}^{\prime}$ of $\mathcal{A}_{2}$,

$$
\Delta_{2}\left(s_{2}\right)\left(s_{2}^{\prime}\right)=\sum_{\sigma_{1} \in S_{1}} \Delta_{1}\left(s_{1}\right)\left(\sigma_{1}\right) \cdot \delta\left(\sigma_{1}\right)\left(s_{2}^{\prime}\right)
$$


Moreover, whenever $\delta\left(\sigma_{1}\right)\left(s_{2}^{\prime}\right)>0, \lambda\left(s_{1}^{\prime}\right)=\lambda\left(s_{2}^{\prime}\right)$. Since $\delta\left(s_{1}^{\prime}\right)$ is a distribution over $S_{2}, \delta\left(s_{1}^{\prime}\right)\left(s_{2}^{\prime}\right)>0$ for at least one state $s_{2}^{\prime}$. Hence $\rho_{0}^{\prime} s_{2}^{\prime}$ is similar to $\rho$, which shows in particular that $w \in F \operatorname{Tr}\left(\mathcal{A}_{2}\right)$.

We additionally show that probabilities coincide:

Proposition 5.4. Let $\mathcal{A}_{1}$ and $\mathcal{A}_{2}$ be PTSs such that $\mathcal{A}_{1}$ refines $\mathcal{A}_{2}$. Then for all $w \in \Sigma^{*}, \mathbf{P}_{\mathcal{A}_{1}}\left(C_{w}\right)=\mathbf{P}_{\mathcal{A}_{2}}\left(C_{w}\right)$.

Since a given word may be produced by several paths, their probabilities should be considered altogether. Hence the proof of the above proposition is not immediate; it is quite technical and can be found in Appendix A.

Existing properties about refinement for PTSs can be retrieved as consequences of the above result. They were for example obtained as a particular case of sub-stochastic refinement in [8]. Although not necessary to prove the main theorem, these results illustrate how constraining refinement between PTSs is.

Recall that a probabilistic bisimulation [9] is a bisimulation that preserves transition probabilities, i.e., a bisimulation relation $\mathcal{R}$ on states such that for any equivalence class $R$ of $\mathcal{R}$, and any two related states $s \mathcal{R} s^{\prime}, \Delta(s)(R)=\Delta\left(s^{\prime}\right)(R)$.

Corollary 5.5 ([8]). Let $\mathcal{A}_{1}$ and $\mathcal{A}_{2}$ be PTSs such that $\mathcal{A}_{1}$ refines $\mathcal{A}_{2}$. Then there exists a probabilistic bisimulation over the union of both PTSs.

Corollary 5.6 ([8]). Let $\mathcal{A}_{1}$ and $\mathcal{A}_{2}$ be PTSs such that $\mathcal{A}_{1}$ refines $\mathcal{A}_{2}$. Then $\mathcal{A}_{2}$ also refines $\mathcal{A}_{1}$.

We are now ready to prove Theorem 5.1:

Proof. Let $\mathcal{A}_{1} \in \underline{\operatorname{sat}}\left(\mathcal{S}_{1}\right)$. By Theorem 5.2 there exists $\mathcal{A}_{2} \in \underline{\operatorname{sat}}\left(\mathcal{S}_{2}\right)$ that is refined by $\mathcal{A}_{1}$. By Proposition 5.4, $\mathbf{P}_{\mathcal{A}_{1}}\left(C_{w}\right)=\mathbf{P}_{\mathcal{A}_{2}}\left(C_{w}\right)$ for every word $w \in F \operatorname{Tr}\left(\mathcal{A}_{1}\right)$. Hence, for any $\omega$-regular (hence measurable) language $\mathcal{L}$, one has $\mathbf{P}_{\mathcal{A}_{1}}(\mathcal{L})=\mathbf{P}_{\mathcal{A}_{2}}(\mathcal{L})$. It is in particular the case for $\mathcal{V}\left(\mathcal{A}_{1}, \mathcal{O}, \varphi\right)=$ $\mathcal{V}\left(\mathcal{A}_{2}, \mathcal{O}, \varphi\right)$. Therefore, $\operatorname{Disc}\left(\mathcal{A}_{1}, \mathcal{O}, \varphi\right)=\operatorname{Disc}\left(\mathcal{A}_{2}, \mathcal{O}, \varphi\right)$. Consequently, the theorem holds.

The result above can now be combined with compositional results on refinement obtained in [12. In particular, since the conjunction of two CMC specifications is the greatest lower bound with respect to weak refinement, and LCMCs are closed under conjunction, we have:

Proposition 5.7. Let $\mathcal{S}_{1}$ and $\mathcal{S}_{2}$ be LCMC specifications. Then Disc $\left(\mathcal{S}_{1} \wedge\right.$ $\left.\mathcal{S}_{2}\right) \leq \min \left(\operatorname{Disc}\left(\mathcal{S}_{1}\right), \operatorname{Disc}\left(\mathcal{S}_{2}\right)\right)$. 


\section{Conclusion}

This work investigates how refinement between probabilistic models impacts the security, modeled as opacity, showing that disclosure is monotonic with respect to refinement when implementations are produced by an adversary through scheduling. We provide 2EXPTIME procedures to compute (1) the worst-case disclosure for a subclass of LCMCs and (2) lower bounds on disclosure for all LCMCs when restricted to polynomial size memory schedulers (in the size of the LCMC).

Directions for future work include computing exact disclosure for LCMCs with modal edges for example by bounding the size of schedulers required to reach the supremal value. We also plan to extend our results to larger sub-classes of CMCs or Parametric IMCs from [12,14. In particular, a question that was left aside is the parallel composition of LCMC, which generates polynomial and possibly non-convex constraints on distributions. Studying the effect of disclosure with respect to this operation would be an interesting (but difficult) task.

\section{References}

1. Clarkson, M.R., Schneider, F.B.: Hyperproperties. Journal of Computer Security 18(6) (September 2010) 1157-1210

2. Alur, R., Černý, P., Zdancewic, S.: Preserving secrecy under refinement. In: Proc. ICALP'06. Volume 4052 of LNCS., Springer (2006) 107-118

3. Mazaré, L.: Decidability of opacity with non-atomic keys. In: Proc. FAST'04. Volume 173 of Intl. Federation for Information Processing., Springer (2005) 71-84

4. Bryans, J.W., Koutny, M., Mazaré, L., Ryan, P.Y.A.: Opacity generalised to transition systems. Intl. Journal of Information Security 7(6) (2008) 421-435

5. Saboori, A., Hadjicostis, C.N.: Current-state opacity formulations in probabilistic finite automata. IEEE Transactions on Automatic Control 59(1) (2014) 120-133

6. Bérard, B., Mullins, J., Sassolas, M.: Quantifying opacity. Mathematical Structures in Computer Science 25(2) (2015) 361-403

7. Bérard, B., Chatterjee, K., Sznajder, N.: Probabilistic Opacity for Markov decision processes. Information Processing Letters 115(1) (2015) 52-59

8. Baier, C., Katoen, J.P., Hermanns, H., Wolf, V.: Comparative branching-time semantics for Markov chains. Information and Computation 200 (2005) 149-214

9. Jonsson, B., Larsen, K.G.: Specification and refinement of probabilistic processes. In: Proc. LICS'91, IEEE Computer Society (1991) 266-277

10. Segala, R.: Modeling and Verification of Randomized Distributed Real-Time Systems. PhD thesis, MIT, Department of Electrical Engineering and Computer Science (1995)

11. Chatterjee, K., Henzinger, T., Sen, K.: Model-checking omega-regular properties of interval Markov chains. In Amadio, R.M., ed.: Proc. FoSSaCS'08. (2008) 302-317

12. Caillaud, B., Delahaye, B., Larsen, K.G., Legay, A., Pedersen, M.L., Wasowski, A.: Constraint Markov chains. Theoretical Computer Science 412(34) (2011) 4373-4404 
13. Benedikt, M., Lenhardt, R., Worrell, J.: LTL model checking of interval Markov chains. In: Proc. TACAS'13. Volume 7795 of LNCS., Springer (2013) 32-46

14. Delahaye, B.: Consistency for parametric interval Markov chains. In André, É., Frehse, G., eds.: Proc. SynCoP'15. Volume 44 of OASICS., Schloss Dagstuhl - LZI (2015) 17-32

15. Bérard, B., Kouchnarenko, O., Mullins, J., Sassolas, M.: Preserving opacity on interval Markov chains under simulation. In Cassandras, C.G., Giua, A., Li, Z., eds.: Proc. of 13th International Workshop on Discrete Event Systems, WODES'16, IEEE (2016) 319-324

16. Billingsley, P.: Probability and Measure. 3rd edn. Wiley (1995)

17. Bérard, B., Mullins, J., Sassolas, M.: Quantifying opacity. In Ciardo, G., Segala, R., eds.: Proc. QEST'10, IEEE Computer Society (September 2010) 263-272

18. Chaum, D.: The dining cryptographers problem: unconditional sender and recipient untraceability. Journal of Cryptology 1 (1988) 65-75

19. Bhargava, M., Palamidessi, C.: Probabilistic Anonymity. In Abadi, M., de Alfaro, L., eds.: Proc. CONCUR'05. Volume 3653 of LNCS. (2005) 171-185

20. Biondi, F., Legay, A., Nielsen, B.F., Wa̧sowski, A.: Maximizing entropy over Markov processes. Journal of Logical and Algebraic Methods in Programming 83(5-6) (2014) 384-399

21. Roos, C., Terlaky, T., Vial, J.P.: Theory and Algorithms for Linear Optimization. An Interior Point Approach. Wiley-Interscience, John Wiley \& Sons Ltd (1997)

22. Sen, K., Viswanathan, M., Agha, G.: Model-checking Markov chains in the presence of uncertainties. In Hermanns, H., Palsberg, J., eds.: Proc. of 12th Intl. Conf. on Tools and Algorithms for the Construction and Analysis of Systems, TACAS'06. Volume 3920 of LNCS., Springer (2006) 394-410

23. Piterman, N.: From Nondeterministic Büchi and Streett Automata to Deterministic Parity Automata. Logical Methods in Computer Science 3(3) (2007)

24. Baier, C., Katoen, J.P.: Principles of Model Checking (Representation and Mind Series). The MIT Press (2008) 


\section{A Proof of Proposition 5.4}

Assume by induction that the proposition holds for every word of length $n$. Let $w \in F \operatorname{Tr}\left(\mathcal{A}_{1}\right)=F \operatorname{Tr}\left(\mathcal{A}_{2}\right)$ (recall Proposition 5.3) of length $n+1$ with $w=w_{0} a$ for some $a \in \Sigma$. A run $\rho^{\prime}$ of $\mathcal{A}_{2}$ that produces $w$ can be assumed to be of the form $\rho^{\prime}=\rho_{0}^{\prime} s_{2}^{\prime}$ with $\operatorname{tr}\left(\rho_{0}^{\prime}\right)=w_{0}$ and $\lambda\left(s_{2}^{\prime}\right)=a$. Then $\mathbf{P}_{\mathcal{A}_{2}}\left(C_{\rho^{\prime}}\right)=\mathbf{P}_{\mathcal{A}_{2}}\left(C_{\rho_{0}^{\prime}}\right) \Delta_{2}\left(s_{2}\right)\left(s_{2}^{\prime}\right)$ where $s_{2}=\operatorname{lst}\left(\rho_{0}^{\prime}\right)$ and hence:

$$
\begin{aligned}
\mathbf{P}_{\mathcal{A}_{2}}\left(C_{w}\right) & =\sum_{\substack{\rho^{\prime} \in F R u n s\left(\mathcal{A}_{2}\right) \\
\operatorname{tr}\left(\rho^{\prime}\right)=w}} \mathbf{P}_{\mathcal{A}_{2}}\left(C_{\rho^{\prime}}\right) \\
& =\sum_{s_{2}, s_{2}^{\prime} \in S_{2}} \sum_{\substack{\rho_{0}^{\prime} \in F \operatorname{Runs}\left(\mathcal{A}_{2}\right) \\
\operatorname{tr}\left(\rho_{0}^{\prime}\right)=w_{0}, \operatorname{lst}\left(\rho_{0}^{\prime}\right)=s_{2}, \operatorname{lst}\left(\rho^{\prime}\right)=s_{2}^{\prime}}} \mathbf{P}_{\mathcal{A}_{2}}\left(C_{\rho_{0}^{\prime}}\right) \cdot \Delta_{2}\left(s_{2}\right)\left(s_{2}^{\prime}\right)
\end{aligned}
$$

Now let $\mathcal{A}_{1}$ s.t. $\mathcal{A}_{2}$ simulates $\mathcal{A}_{1}$ then, as

$$
\sum_{\substack{\left.\rho_{0} \in F R u n s \\
\rho_{0} \sim \rho_{0}^{\prime} \\
\operatorname{lst}\left(\rho_{0}\right)=s_{1}\right)}} \mu_{\rho_{0}^{\prime}}\left(\rho_{0}\right)=\sum_{s_{1} \in S_{1}} \sum_{\begin{array}{c}
\rho_{0} \in F R u n s \\
\rho_{0} \sim \rho_{0}^{\prime} \\
\operatorname{lst}\left(\rho_{0}\right)=s_{1}
\end{array}} \mu_{\rho_{0}^{\prime}}\left(\rho_{0}\right)=1
$$

we get:

$$
\begin{aligned}
& \mathbf{P}_{\mathcal{A}_{2}}\left(C_{w}\right)=\sum_{s_{2}, s_{2}^{\prime} \in S_{2}} \sum_{\substack{\left.\rho_{0}^{\prime} \in F R u n s \\
\operatorname{tr}\left(\rho_{0}^{\prime}\right)=w_{0}\right) \\
\operatorname{lst}\left(\rho_{0}^{\prime}\right)=s_{2}, \operatorname{lst}\left(\rho^{\prime}\right)=s_{2}^{\prime}}} \mathbf{P}_{\mathcal{A}_{2}}\left(C_{\rho_{0}^{\prime}}\right) \cdot \Delta_{2}\left(s_{2}\right)\left(s_{2}^{\prime}\right) \cdot \sum_{s_{1} \in S_{1}} \sum_{\substack{\left.\rho_{0} \in F R u n s \\
\rho_{0} \sim \rho_{0}^{\prime} \\
\operatorname{lst}\left(\rho_{0}\right)=s_{1}\right)}} \mu_{\rho_{0}^{\prime}}\left(\rho_{0}\right) \\
& =\sum_{\substack{s_{1} \in S_{1} \\
s_{2}, s_{2}^{\prime} \in S_{2}}} \sum_{\substack{\left.\rho_{0}^{\prime} \in F R u n s \\
\operatorname{tr}\left(\rho_{0}^{\prime}\right)=w_{0}\right) \\
\operatorname{lst}\left(\rho_{0}^{\prime}\right)=s_{2}, \operatorname{lst}\left(\rho^{\prime}\right)=s_{2}^{\prime}}} \mathbf{P}_{\mathcal{A}_{2}}\left(C_{\rho_{0}^{\prime}}\right) \cdot \Delta_{2}\left(s_{2}\right)\left(s_{2}^{\prime}\right) \sum_{\substack{\rho_{0} \in F R u n s \\
\rho_{0} \sim \rho_{0}^{\prime} \\
\operatorname{lst}\left(\rho_{0}\right)=s_{1}}} \mu_{\rho_{0}^{\prime}}\left(\rho_{0}\right)
\end{aligned}
$$

Since the terms are null if it is not the case that $s_{1} \mathcal{R} s_{2}$, we have:

$$
\begin{aligned}
\mathbf{P}_{\mathcal{A}_{2}}\left(C_{w}\right)= & \sum_{\substack{s_{1} \in S_{1} \\
s_{2}, s_{2}^{\prime} \in S_{2}}} \sum_{\substack{\rho_{0}^{\prime} \in F R u n s\left(\mathcal{A}_{2}\right) \\
\operatorname{tr}\left(\rho_{0}^{\prime}\right)=w_{0}, \operatorname{lst}\left(\rho_{0}^{\prime}\right)=s_{2}, \operatorname{lst}\left(\rho^{\prime}\right)=s_{2}^{\prime}}} \mathbf{P}_{\mathcal{A}_{2}}\left(C_{\rho_{0}^{\prime}}\right) \cdot \\
& \left(\sum_{s_{1}^{\prime} \in S_{1}} \Delta_{1}\left(s_{1}\right)\left(s_{1}^{\prime}\right) \cdot \delta_{s_{1}, s_{2}}\left(s_{1}^{\prime}\right)\left(s_{2}^{\prime}\right) \sum_{\substack{\rho_{0} \in F R u n s\left(\mathcal{A}_{1}\right) \\
\rho_{0} \sim \rho_{0}^{\prime} \\
\operatorname{lst}\left(\rho_{0}\right)=s_{1}}} \mu_{\rho_{0}^{\prime}}\left(\rho_{0}\right)\right)
\end{aligned}
$$


And since the terms are null if $\lambda\left(s_{1}^{\prime}\right) \neq \lambda\left(s_{2}^{\prime}\right)=\lambda(\operatorname{lst}(\rho))$ :

$$
\begin{aligned}
& \mathbf{P}_{\mathcal{A}_{2}}\left(C_{w}\right)=\sum_{\substack{s_{1} \in S_{1} \\
s_{2}, s_{2}^{\prime} \in S_{2} \\
\operatorname{lst}\left(\rho_{0}^{\prime}\right)=s_{2}, \operatorname{lst}\left(\rho^{\prime}\right)=s_{2}^{\prime} \in F R u n s\left(\mathcal{A}_{2}\right)}} \mathbf{P}_{\mathcal{A}_{2}}\left(C_{\rho_{0}^{\prime}}\right) \sum_{\substack{\left.\operatorname{tr}_{0}^{\prime}\right)=w_{0} \\
s_{1}^{\prime} \in S_{1} \\
\lambda\left(s_{1}\right)=\lambda(\operatorname{lst}(\rho))}} \Delta_{1}\left(s_{1}\right)\left(s_{1}^{\prime}\right) \cdot \\
& \left(\begin{array}{c}
\delta_{s_{1}, s_{2}}\left(s_{1}^{\prime}\right)\left(s_{2}^{\prime}\right) \sum_{\substack{\rho_{0} \in F R u n s \\
\rho_{0} \sim \rho_{0}^{\prime} \\
\operatorname{lst}\left(\rho_{0}\right)=s_{1}}} \mu_{\rho_{0}^{\prime}}\left(\rho_{0}\right) \\
\left.\cos ^{\prime}\right)
\end{array}\right) \\
& =\sum_{s_{1} \in S_{1}} \sum_{\substack{s_{1}^{\prime} \in S_{1} \\
\lambda\left(s_{1}\right)=\lambda(\operatorname{lst}(\rho))}} \sum_{\substack{s_{2} \in S_{2} \\
s_{2}^{\prime} \in S_{2}}} \delta_{s_{1}, s_{2}}\left(s_{1}^{\prime}\right)\left(s_{2}^{\prime}\right) .
\end{aligned}
$$

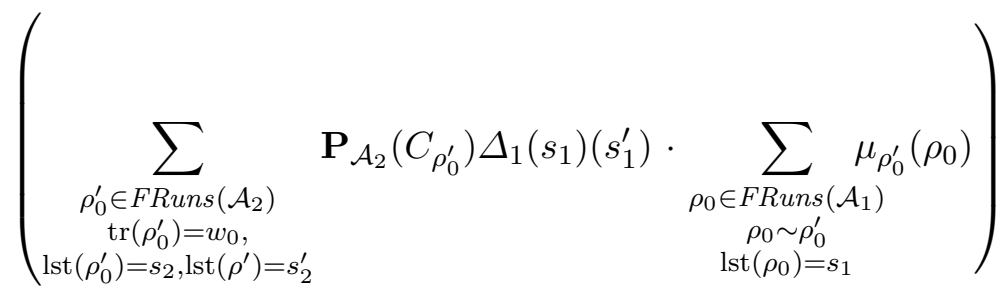

Since $\sum_{s_{2}^{\prime} \in S_{2}} \delta_{s_{1}, s_{2}}\left(s_{1}^{\prime}\right)\left(s_{2}^{\prime}\right)=1$ :

$$
\begin{aligned}
& \mathbf{P}_{\mathcal{A}_{2}}\left(C_{w}\right)=\sum_{s_{1} \in S_{1}} \sum_{\substack{s_{1}^{\prime} \in S_{1} \\
\lambda\left(s_{1}\right)=\lambda(\operatorname{lst}(\rho))}} \sum_{\substack{s_{2} \in S_{2} \\
\rho_{0}^{\prime} \in F R u n s\left(\mathcal{A}_{2}\right) \\
\operatorname{tr}\left(\rho_{0}^{\prime}\right)=w_{0}, \operatorname{lst}\left(\rho_{0}^{\prime}\right)=s_{2}}} \mathbf{P}_{\mathcal{A}_{2}}\left(C_{\rho_{0}^{\prime}}\right) \cdot \Delta_{1}\left(s_{1}\right)\left(s_{1}^{\prime}\right) \sum_{\substack{\left.\rho_{0} \in F R u n s \\
\rho_{0} \sim \rho_{0}^{\prime} \\
\operatorname{lst}\left(\rho_{0}\right)=s_{1}\right)}} \mu_{\rho_{0}^{\prime}}\left(\rho_{0}\right) \\
& =\sum_{\substack{s_{1} \in S_{1} \\
\lambda\left(s_{1}\right)=\lambda(\operatorname{lst}(\rho))}} \sum_{\substack{\left.\rho_{0}^{\prime} \in S_{1} \in R u n s \\
\operatorname{tr}\left(\rho_{0}^{\prime}\right)=w_{0}\right)}} \mathbf{P}_{\mathcal{A}_{2}}\left(C_{\rho_{0}^{\prime}}\right) \cdot \Delta_{1}\left(s_{1}\right)\left(s^{\prime}\right) \sum_{\substack{\rho_{0} \in F R u n s \\
\rho_{0} \sim \rho_{0}^{\prime} \\
\operatorname{lst}\left(\rho_{0}\right)=s_{1}}} \mu_{\rho_{0}^{\prime}}\left(\rho_{0}\right) \\
& \left.=\sum_{\substack{s_{1} \in S_{1} \\
\lambda\left(s_{1}\right)=\lambda(\operatorname{lst}(\rho))}} \sum_{\substack{s_{1}^{\prime} \in S_{1} \\
\lambda}} \Delta_{1}\right)\left(s_{1}^{\prime}\right) \cdot \sum_{\substack{\left.\rho_{0}^{\prime} \in F R u n s \\
\operatorname{tr}\left(\rho_{0}^{\prime}\right)=\mathcal{A}_{0}\right)}} \sum_{\substack{\left.\rho_{0} \in F R u n s \\
\rho_{0} \sim \mathcal{A}_{1}^{\prime}\right) \\
\operatorname{lst}\left(\rho_{0}\right)=s_{1}}} \mathbf{P}_{\mathcal{A}_{2}}\left(C_{\rho_{0}^{\prime}}\right) \mu_{\rho_{0}^{\prime}}\left(\rho_{0}\right) \\
& =\sum_{s_{1} \in S_{1}} \sum_{\substack{s_{1}^{\prime} \in S_{1} \\
\lambda\left(s_{1}\right)=\lambda(\operatorname{lst}(\rho))}} \Delta_{1}\left(s_{1}\right)\left(s_{1}^{\prime}\right) \sum_{\substack{\rho_{0}^{\prime} \in F R u n s\left(\mathcal{A}_{2}\right) \\
\operatorname{tr}\left(\rho_{0}^{\prime}\right)=w_{0}}} \cdot \sum_{\substack{\left.\rho_{0} \in F R u n s \\
\rho_{0} \sim \rho_{0}^{\prime} \\
\operatorname{lst}\left(\rho_{0}\right)=s_{1}\right)}} \mathbf{P}_{\mathcal{A}_{2}}\left(C_{\rho_{0}^{\prime}}\right) \frac{\mathbf{P}_{\mathcal{A}_{1}}\left(C_{\rho_{0}}\right)}{\mathbf{P}_{\mathcal{A}_{1}}\left(\operatorname{sim}\left(\rho_{0}^{\prime}\right)\right)}
\end{aligned}
$$




$$
\begin{aligned}
& \left.=\sum_{\substack{s_{1} \in S_{1} \\
\lambda\left(s_{1}\right)=\lambda(\operatorname{lst}(\rho))}} \sum_{\substack{s_{1}^{\prime} \in S_{1} \\
s_{1}}} s_{1}\right)\left(s_{1}^{\prime}\right) \sum_{\substack{\rho_{0} \in F R u n s\left(\mathcal{A}_{1}\right) \\
\operatorname{tr}\left(\rho_{0}\right)=w_{0} \\
\operatorname{lst}\left(\rho_{0}\right)=s_{1}}} \frac{\mathbf{P}_{\mathcal{A}_{1}}\left(C_{\rho_{0}}\right)}{\mathbf{P}_{\mathcal{A}_{1}}\left(\operatorname{sim}\left(\rho_{0}^{\prime}\right)\right)} \sum_{\substack{\left.\rho_{0}^{\prime} \in F R u n s \\
\operatorname{tr}\left(\rho_{0}^{\prime}\right)=w_{0}\right)}} \mathbf{P}_{\mathcal{A}_{2}}\left(C_{\rho_{0}^{\prime}}\right)
\end{aligned}
$$

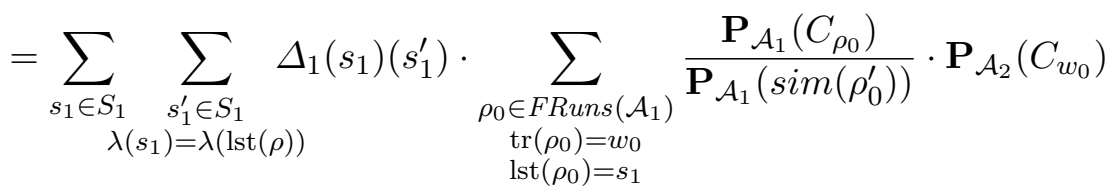

By induction hypothesis, $\mathbf{P}_{\mathcal{A}_{2}}\left(C_{w_{0}}\right)=\mathbf{P}_{\mathcal{A}_{1}}\left(C_{w_{0}}\right)$ hence:

$$
\mathbf{P}_{\mathcal{A}_{2}}\left(C_{w}\right)=\sum_{s_{1} \in S_{1}} \sum_{\substack{s_{1}^{\prime} \in S_{1} \\ \lambda\left(s_{1}\right)=\lambda(\operatorname{lst}(\rho))}} \Delta_{1}\left(s_{1}\right)\left(s_{1}^{\prime}\right) \cdot \sum_{\substack{\rho_{0} \in F R u n s\left(\mathcal{A}_{1}\right) \\ \operatorname{tr}\left(\rho_{0}\right)=w_{0} \\ \operatorname{lst}\left(\rho_{0}\right)=s_{1}}} \frac{\mathbf{P}_{\mathcal{A}_{1}}\left(C_{\rho_{0}}\right)}{\mathbf{P}_{\mathcal{A}_{1}}\left(\operatorname{sim}\left(\rho_{0}^{\prime}\right)\right)} \cdot \mathbf{P}_{\mathcal{A}_{1}}\left(C_{w_{0}}\right)
$$

and since $\mathbf{P}_{\mathcal{A}_{1}}\left(\operatorname{sim}\left(\rho_{0}^{\prime}\right)\right)=\mathbf{P}_{\mathcal{A}_{1}}\left(C_{w_{0}}\right)$ :

$$
\begin{aligned}
\mathbf{P}_{\mathcal{A}_{2}}\left(C_{w}\right) & =\sum_{s_{1} \in S_{1}} \sum_{\substack{s_{1}^{\prime} \in S_{1} \\
\lambda\left(s_{1}\right)=\lambda(\operatorname{lst}(\rho))}} \Delta_{1}\left(s_{1}\right)\left(s_{1}^{\prime}\right) \cdot \sum_{\substack{\left.\rho_{0} \in F R u n s \\
\operatorname{tr}\left(\rho_{0}\right)=w_{0}\right) \\
\operatorname{lst}\left(\rho_{0}\right)=s_{1}}} \mathbf{P}_{\mathcal{A}_{1}}\left(C_{\rho_{0}}\right) \\
& =\sum_{s_{1} \in S_{1}} \sum_{\substack{\left.\rho_{0} \in F R u n s \\
\operatorname{tr}\left(\rho_{0}\right)=w_{0}\right) \\
\operatorname{lst}\left(\rho_{0}\right)=s_{1}}} \mathbf{P}_{\mathcal{A}_{1}}\left(C_{\rho_{0}}\right) \cdot \sum_{\substack{\left.\lambda_{1}^{\prime} \in S_{1} \\
s_{1}\right)=\lambda(\operatorname{lst}(\rho))}} \Delta_{1}\left(s_{1}\right)\left(s_{1}^{\prime}\right) \\
& =\sum_{\substack{\rho_{0} \in F \operatorname{Runs}\left(\mathcal{A}_{1}\right) \\
\operatorname{tr}\left(\rho_{0}\right)=w_{0}}} \mathbf{P}_{\mathcal{A}_{1}}\left(C_{\rho_{0}}\right) \Delta_{1}\left(\operatorname{lst}\left(\rho_{0}\right)\right)(\operatorname{lst}(\rho))=\mathbf{P}_{\mathcal{A}_{1}}\left(C_{w}\right)
\end{aligned}
$$

